\title{
An Innovative Solar-Powered Natural Gas-Based Compressed Air Energy Storage System Integrated with a Liquefied Air Power Cycle
}

\author{
Shayan Sadeghi ${ }^{1}$, Samane Ghandehariun ${ }^{1}$, Behnaz Rezaie ${ }^{2}$, Nader Javani ${ }^{3}$ \\ ${ }^{1}$ Sustainable Energy Research Group (SERG), School of Mechanical Engineering, Iran University of \\ Science and Technology, Tehran, Iran \\ ${ }^{2}$ Department of Mechanical Engineering, College of Engineering, University of Idaho, 875 Perimeter Dr., \\ Moscow, ID 83844-0902, USA \\ ${ }^{3}$ Faculty of Mechanical Engineering, Yildiz Technical University, 34349, Istanbul, Turkey
}

\begin{abstract}
A novel solar-based compressed air energy storage system is developed and analyzed in this paper. The integrated system includes a multi-stage air compression unit, thermal oil loop, multi-stage gas turbine unit, high-temperature molten salt-based solar power tower unit, liquefied air power cycle, thermoelectric generator, and liquefied natural gas (LNG) regasification unit. A eutectic mixture of carbonate salt is used for thermal energy storage in the solar subsystem. Energy, exergy, and economic analyses are performed to evaluate the performance of the proposed system. A parametric study investigates the effects of important parameters on the performance of the integrated system. The proposed system stores $356 \mathrm{MWh}$ of grid electricity during the charging mode. Considering all the available energy sources, the energy output of the integrated system is 797.6 MWh. Energy and exergy efficiencies of the integrated system are $55.3 \%$ and $46.4 \%$, respectively. The results show that the highest contributor to the overall exergy destruction rate of the integrated system is the combustion chamber unit. Finally, levelized cost of electricity is evaluated as $12.4 \phi / \mathrm{kWh}$.
\end{abstract}

Keywords: Compressed air energy storage; Solar energy; Economic analysis; Energy and exergy analyses; LNG regasification; Thermal energy storage

Nomenclature

A

Ex

ex

$\mathrm{h}$

I

$\dot{m}$
Area $\left(\mathrm{m}^{2}\right)$

Exergy (W)

Specific exergy $(\mathrm{kJ} / \mathrm{kg})$

Specific enthalpy $(\mathrm{kJ} / \mathrm{kg})$

Irradiance $\left(\mathrm{W} / \mathrm{m}^{2}\right)$

Mass flow rate $(\mathrm{kg} / \mathrm{s})$ 


\begin{tabular}{|c|c|}
\hline $\mathrm{P}$ & Pressure $(\mathrm{kPa})$ \\
\hline$\dot{Q}$ & Heat transfer rate $(\mathrm{W})$ \\
\hline $\mathrm{t}$ & Time \\
\hline $\mathrm{T}$ & Temperature $(\mathrm{K})$ \\
\hline$\dot{W}$ & Power (W) \\
\hline $\mathrm{y}_{\mathrm{x}}$ & Coefficient of specie $\mathrm{x}$ \\
\hline \multicolumn{2}{|c|}{ Subscripts } \\
\hline $\mathrm{ch}$ & Chemical exergy \\
\hline $\mathrm{D}$ & Destruction \\
\hline $\mathrm{h}$ & Heliostats \\
\hline ke & Kinetic exergy \\
\hline pe & Potential exergy \\
\hline $\mathrm{ph}$ & Physical exergy \\
\hline \multicolumn{2}{|c|}{ Greek symbols } \\
\hline$\eta_{\mathrm{I}}$ & Energy efficiency \\
\hline$\eta_{\text {II }}$ & Exergy efficiency \\
\hline $\bar{\lambda}$ & Molar fuel to air ratio \\
\hline$\Delta$ & Difference \\
\hline$\varepsilon$ & Normalized carbon dioxide emissions $\left(\mathrm{kg} \mathrm{CO}_{2} / \mathrm{MWh}\right)$ \\
\hline \multicolumn{2}{|c|}{ Abbreviations } \\
\hline CAES & Compressed air energy storage \\
\hline CCHP & Combined cooling, heating, and power \\
\hline GTIT & Gas turbine inlet temperature \\
\hline PEC & Purchase equipment cost \\
\hline TRR & Total revenue requirement \\
\hline
\end{tabular}

\section{Introduction}

With the exhaustion of fossil fuel resources and aggravation of global energy demand, the utilization of renewable energies to achieve sustainability has become an important subject of research and development in the modern era [1]. One of the main challenges associated with renewable energies is their intermittent nature [2]. To deal with this obstacle and to manage the outgrowing problems of high energy demand, it is necessary to use energy storage systems that store energy when it is not needed and use it later when the demand is high [3].

Energy may be stored in the form of mechanical, chemical, or thermal energy [4]. Compressed air energy storage (CAES), as a mechanical energy storage method, is one of the most promising methods to resolve the intermittent issues of renewable energies [5]. This method possesses 
various advantages including technological maturity, availability of equipment, low investment cost, and high energy storage capacity [6,7].

CAES systems store energy through a series of air compressors to pressurize and store air in underground reservoirs such as salt caverns or high-pressure tanks above ground. When energy is required, the pressurized air flows through a series of expansion units in order to generate power [8]. To increase the system efficiency, it is convenient to use the heat generated in the compression process to heat up the air before entering the expanders [8]. Liu et al. [9] investigated the CAES system through an advanced exergetic analysis. Their results indicated that the round-trip and exergy efficiencies of the cycle were $47.4 \%$ and $41.2 \%$, respectively. Advanced exergetic analysis showed that $34.6 \mathrm{MW}$ of the total exergy destruction of the system is not related to the irreversibilities, and therefore, it is avoidable. More specifically, intercooler, aftercooler, and combustion chamber account for around 58\% of the avoidable exergy destruction in the system [9]. In another study, Krawczyk et al. [10] investigated two compressed air energy storage systems including a conventional diabatic CAES and a liquefied air energy storage (LAES). The roundtrip efficiency of the CAES and LAES systems were reported as $39.77 \%$ and $55.2 \%$, respectively [10]. Peng et al. [11] proposed and analyzed an adiabatic CAES system coupled with a packed bed thermal energy storage system and reported an overall efficiency of 56.74\% [11]. In another comparative study, Liu and Wang [12] investigated two different adiabatic CAES systems for energy storage. One of which was a conventional CAES system and the other was a modified version of CAES that used a pneumatic motor, heat accumulator, and preheater. The results indicated that the modified version had a round trip efficiency of $66.9 \%$ which is $13 \%$ higher than that of the conventional CAES system [12]. Coupling of the CAES system with the Kalina cycle was proposed by Zhao et al. [13]. Kalina cycle is used to recover the waste heat of the CAES system and a round trip efficiency of $47.64 \%$ was reported [13]. In another study, Szablowski et al. [14] investigated an adiabatic CAES system in terms of energetic and exergetic perspectives. The round-trip efficiency of their proposed cycle was 50\% [14]. A transient thermodynamic model for an underwater CAES system was developed and investigated by Carriveau et al. [15]. The results indicated that the heat exchangers had the highest rates of exergy destruction among other system components considering the steady-state condition. Under transient condition, the highest exergy destruction contributors are air compressors with a value of $40 \%$ of the total exergy destruction of the system [15].

For increasing the performance of gas turbines (GT) or CAES systems, one convenient way is to use natural gas to achieve higher temperatures for the inlet of the gas turbines [16]. As the outlet temperature of the gas turbine is high, they can be coupled with a bottoming cycle to increase the performance of the system [16]. A commonly used combined power cycle utilizes a steam Rankine cycle coupled with the gas turbine units for this mean [17]. Natural gas is mostly liquefied in order to be transported more efficiently in large distances. When it is needed again, it is regasified using cryogenic pumps, heat exchangers, and expanders. The cold waste heat of this process can be used as a heat sink of many power generation processes to yield additional power and increase the cycle performance [18]. Several working fluids are suitable for this purpose [19]. As an example of the compressed gas energy storage, the feasibility of compressed carbon dioxide energy storage (CCES) integrated with low-temperature thermal energy storage has been studied in the literature [20] in which the results show the feasibility of the cycle to be used in cogeneration systems. For the assessment of compressed air energy storage systems, the heat transfer mechanism and heat 
losses from the storage tank or cavern should be considered. An experimental and theoretical study has been conducted for a high-pressure compressed air tank storage system in which the experimental data was used to assess the wall and air temperatures in the pressurized storage tank of 100 bar. The results showed that the tank wall should be taken into account in the thermal analysis of CAES systems [21]. In another study, a CAES system integrated with a biomass-driven power plant with thermal energy storage was studied and the effects of heat loss in the cavern and the temperature of the soil around the cavern on the performance of the system were investigated [22].

Air, as a free and non-toxic working fluid, is a great choice for this mean. Air with a composition of $75.57 \%$ nitrogen, $23.16 \%$ oxygen, and $1.27 \%$, has a critical temperature of about $-140{ }^{\circ} \mathrm{C}$ while the temperature of $\mathrm{LNG}$ at atmospheric pressure is about $-160^{\circ} \mathrm{C}$. Such temperature difference is enough to liquefy air in the cryogenic condenser of a liquefied air power cycle. The technology of such power systems is similar to those used in the LAES systems [23].

To improve the thermal efficiency and reliability of a CAES system various thermodynamic cycle can be used and integrated with renewable energy-based systems. Wang et al. [24] proposed and analyzed an underwater CAES system driven by marine renewable energies for an island using energy and exergy thermodynamic analyses. Cold and hot thermal oil loops were utilized in their studies to improve the system performance. Both energy and exergy round-trip efficiencies are evaluated as 58\% for their proposed system [24]. In another study, Wang et al. [25] investigated the integration of ORC with an adiabatic CAES system to produce power, heating, and cooling. Four different configurations of CAES systems were proposed and analyzed in their study. These configurations differed from the number of expansion stages, thermal oil loops, and heat exchanger configurations. Their results indicated that the energy efficiency of these systems was between $52 \%$ to 59.6\% [25]. Hao et al. [26] proposed a novel energy storage system similar to a conventional CAES system that utilizes carbon dioxide as its working fluid, and also, used a heat pump to eliminate the use of natural gas in the discharging mode. Energetic and exergetic efficiencies of the system were obtained as $58.4 \%$ and $67.9 \%$, respectively [26]. An integrated wind/solar-based CAES system was investigated by Ji et al. [27]. The round-trip and exergy efficiencies of the proposed system were $61.2 \%$ and $65.4 \%$, respectively [27]. Mohammadi and Mehrpooya [28] investigated the coupling of a solar dish with the CAES system. The system consumed $152 \mathrm{~kW}$ during charging mode for compression of the air and by using the solar heat to increase the inlet temperature of the gas turbine produced $228 \mathrm{~kW}$ during discharging mode. Exergy efficiency of $53.4 \%$ and energy efficiency of $76.5 \%$ were reported [28]. Yang et al. [29] proposed an integration of a solar CAES system with combined heating, cooling, and power system for a hotel building in south China. The integrated system considered in their study included solar collector assembly, gas turbine cycle, absorption chiller, heat recovery steam generator unit, and CAES system. Solar collectors were utilized to enhance the temperature of the air before entering the gas turbine to improve the system efficiency. It was concluded that their proposed system enhanced the efficiency of previous systems by $1.01 \%$. Under different design conditions, the energy efficiency of their integrated system varied between 6-39\% [29].

A novel integration of CAES system with turbocharger and fuel cell subsystems for the production of hot water and electricity is proposed and analyzed thermodynamically by Roushenas et al. [30]. Energetic and exergetic efficiencies were 58\% and 78\%, respectively [30]. The thermodynamic performance of an integrated wind-powered CAES system coupled with an 
absorption refrigeration cycle and an ORC for the production of cooling, electricity, and heating is investigated by Mohammadi et al. [31]. The energy efficiency of their system was 53.9\% [31]. A comparison of selected past studies is presented in Table 1.

Table 1 A comparison of past studies on the CAES systems.

\begin{tabular}{|c|c|c|c|c|c|c|}
\hline Study & Year & $\begin{array}{l}\text { Renewable } \\
\text { energy source }\end{array}$ & System outputs & $\begin{array}{l}\text { Energy } \\
\text { efficiency } \\
(\%)\end{array}$ & $\begin{array}{l}\text { Exergy } \\
\text { efficiency } \\
(\%)\end{array}$ & Reference \\
\hline Wang et al. & 2019 & Marine energy & Power & 58 & 58 & [24] \\
\hline Wang et al. & 2019 & - & $\begin{array}{l}\text { Power, heating, } \\
\text { and cooling }\end{array}$ & $52-59.6$ & - & [25] \\
\hline Hao et al. & 2020 & - & Power & 58.4 & 67.9 & [26] \\
\hline Ji et al. & 2017 & Solar and wind & $\begin{array}{l}\text { Power and hot } \\
\text { water }\end{array}$ & 61.2 & 65.4 & [27] \\
\hline $\begin{array}{l}\text { Mohammadi } \\
\text { et al. }\end{array}$ & 2016 & Solar & Power & 76.5 & 53.4 & [28] \\
\hline Yang et al. & 2017 & Solar & $\begin{array}{l}\text { Power, heating, } \\
\text { and cooling }\end{array}$ & 6-39 & - & [29] \\
\hline $\begin{array}{l}\text { Roushenas } \\
\text { et al. }\end{array}$ & 2020 & - & $\begin{array}{l}\text { Power and hot } \\
\text { water }\end{array}$ & 58 & 78 & [30] \\
\hline $\begin{array}{l}\text { Mohammadi } \\
\text { et al. }\end{array}$ & 2017 & Wind & $\begin{array}{l}\text { Power and hot } \\
\text { water }\end{array}$ & 53.9 & - & [31] \\
\hline
\end{tabular}

As mentioned earlier, there have been afforts to improve the performance of the conventional CAES systems by utilizing modified configurations or integrating with other thermodynamic cycles and renewable energy-based systems. However, coupling of CAES with liquefied air power cycle, utilization of LNG regasification waste heat, utilization of thermoelectric generators (TEG), and utilization of solar power towers has not been studided in the past.

The main objective of the current study is to conduct a thermodynamic analysis using energy and exergy methods for a novel integrated CAES system that uses solar energy, LNG regasification waste heat, air power cycle, and TEG for the cogeneration of cooling and electricity. A hightemperature carbonate molten salt is used as the working fluid of the solar power tower unit to heat the air before entering the expanders in the discharging phase of the system. Natural gas is also used in this hybrid system to increase the system performance and power yield. The waste heat of the exhaust gas is used to drive an air power cycle and a TEG unit. A parametric study is carried out to investigate the effects of varying major design parameters on the system performance. The results presented in this paper enable types, locations, and magnitudes of losses to be determined to reduce sources of inefficiency in the proposed system. The main features of the paper are as follows:

- A cogeneration CAES system is proposed for the production of electricity and cooling.

- Solar and natural gas are used in the discharging phase of the system to increase the system performance and sustainability.

- High-temperature molten salt is utilized as the heat transfer fluid and thermal energy storage medium of the solar power tower unit. 
- The waste heat of the exhaust gas is used to drive an air power cycle and TEG unit.

- The waste heat of the LNG regasification unit is used to improve the performance of the integrated system.

- A thermal oil loop is utilized to capture the heat of the compression process and use it in the discharge phase of the plant.

\section{Proposed System Description}

Schematic of the diabatic solar-assisted CAES system is presented in Fig. 1. The integrated system comprises an air compression unit, thermal oil loop, solar power tower with thermal energy storage, gas turbine with a combustor, thermoelectric generator, liquefied air power cycle, and LNG regasification unit.

During charging mode, the air is pressurized by a double-stage air compressor to store the energy. Thermal oil leaving the cold tank is used in the intercooler and aftercooler units to recover the heat produced during the compression process. The recovered heat is then used during the discharging mode to increase the cycle performance. Compressed air is stored in the salt cavern at near ambient temperature. The heated thermal oil is stored in the hot tank. Base on past studies, a pressure regulating valve at the outlet of the salt cavern was not considered in this paper [28,29,31]. During the discharging mode, the air flowing out of the cavern is firstly heated up in heater 1 by the thermal oil, and then in heater 2 by the high-temperature molten salt before entering the first stage of the gas turbine unit to generate power. High-temperature molten salt, which is a eutectic mixture of $\mathrm{Li}_{2} \mathrm{CO}_{3}(32.1 \%)-\mathrm{Na}_{2} \mathrm{CO}_{3}(33.4 \%)-\mathrm{K}_{2} \mathrm{CO}_{3}(34.5 \%)$, is used for thermal energy storage and also, as the heat transfer fluid in the solar power tower [32]. After the first expansion process, the air enters the combustion chamber unit where natural gas is used to increase the temperature of the air before the next expansion process. For the base case, exhaust gases with a temperature of $1100{ }^{\circ} \mathrm{C}$ enter the second stage of the gas turbine unit to produce power. Exhaust gases of the second stage pass through the heater 3 to provide the required heat of the liquefied air power cycle. As the temperature of the flue gas leaving the heater 3 is still high, a thermo-electric generator is utilized to recover the remaining thermal energy of the flue gas before discharging it to the environment at $150{ }^{\circ} \mathrm{C}$.

In the liquefied air power cycle, air is pressurized by the pump and enters two sequential heat exchangers where it is superheated before entering the turbine to generate power. The expanded air exits the turbine at a lower temperature $\left(455^{\circ} \mathrm{C}\right.$ for the base case) and enters the recuperator where rejects heat to the pressurized cold air. The air at the exit of the recuperator, state 37 is a very low temperature of $-109.6{ }^{\circ} \mathrm{C}$. At the same time, $\mathrm{LNG}$ exiting the tank is pressurized by the LNG pump for the regasification process. The temperature of the liquefied air power cycle condenser is a function of LNG temperature at the outlet of the pump. For the LNG regasification process, the natural gas must be heated up before entering the expander to increase the power yield of the regasification process. Therefore, LNG then goes through heater 4 to be heated up by the thermal oil. It then enters heater 5 which produces cooling before entering the natural gas expander. The natural gas leaves the system to be transported by pipeline. 


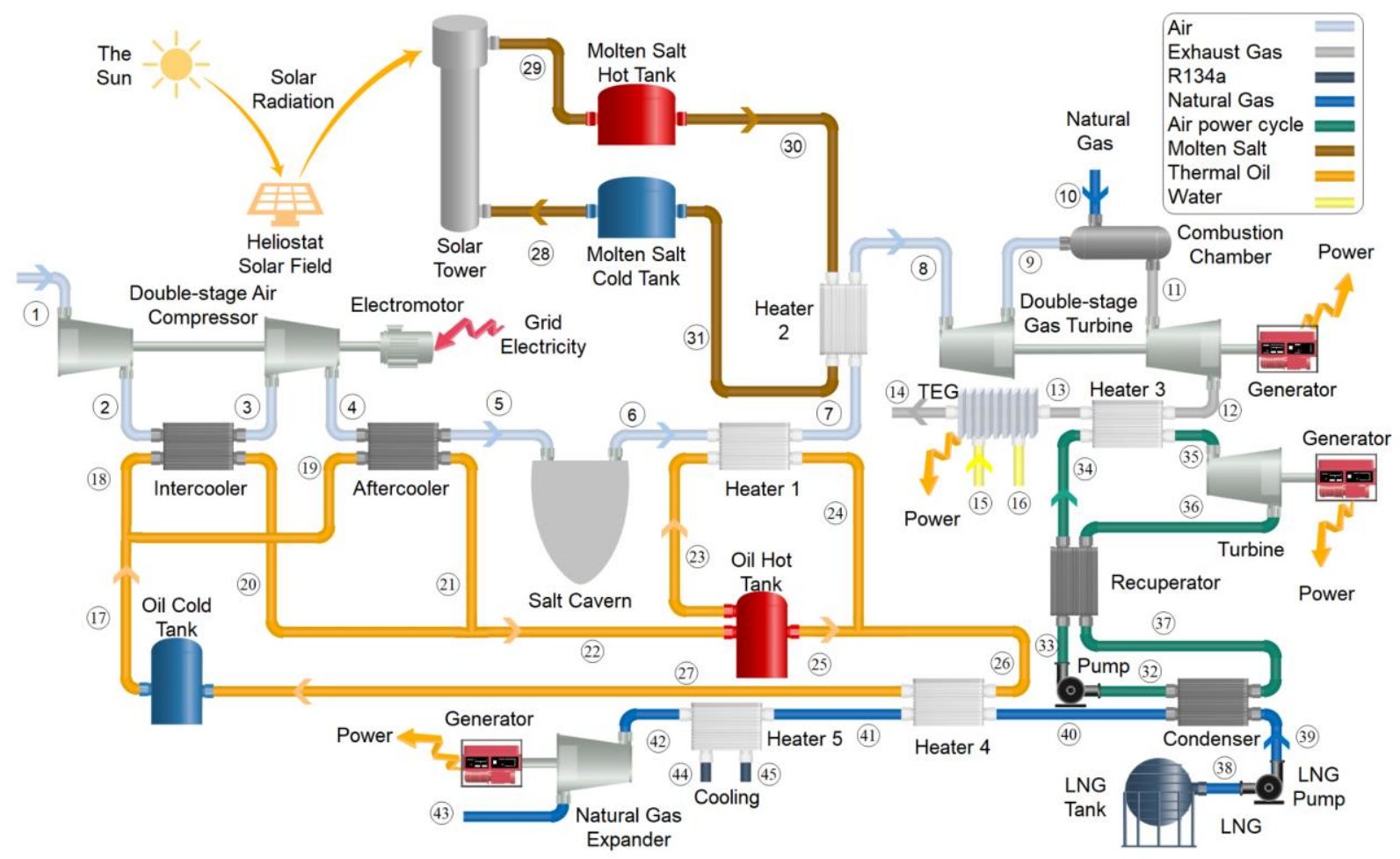

Figure 1 Schematic of the solar-assisted CAES system.

\section{Methodology and Analysis}

As mentioned earlier, energy and exergy approaches are used for the thermodynamic analysis in this study [33]. MATLAB software integrated with REFPROP 9 library is utilized to develop a thermodynamic model used for the integrated system. The conservations of mass and energy for the steady-state condition are presented respectively as follows:

$$
\begin{aligned}
& \sum \dot{\mathrm{m}}_{\mathrm{i}}-\sum \dot{\mathrm{m}}_{\mathrm{e}}=0 \\
& \dot{\mathrm{Q}}-\dot{\mathrm{W}}=\sum(\dot{\mathrm{m} h})_{\mathrm{e}}-\sum(\dot{\mathrm{m} h})_{\mathrm{i}}
\end{aligned}
$$

Exergy rate balance at steady-state is expressed by:

$$
\dot{E} x_{W}-\dot{E x} x_{Q}=\sum\left(\dot{m e x}_{f}\right)_{i}-\sum\left(\dot{m e x}_{f}\right)_{e}-\dot{E} x_{D}
$$

where $\dot{\mathrm{Ex}}_{\mathrm{W}}$ and $\dot{\mathrm{Ex}} \mathrm{Q}_{\mathrm{Q}}$ are rates of exergy transfer accompanying heat transfer and work, respectively, and $\mathrm{ex}_{\mathrm{f}}$ is the total flow exergy:

$$
\dot{\mathrm{Ex}_{\mathrm{Q}}}=\left(1-\frac{\mathrm{T}_{0}}{\mathrm{~T}_{\mathrm{i}}}\right) \times \dot{\mathrm{Q}_{1}}
$$




$$
\begin{aligned}
& \dot{\mathrm{Ex}} \mathrm{W}_{\mathrm{W}}=\dot{\mathrm{W}} \\
& \mathrm{ex}_{\mathrm{f}}=\mathrm{ex}_{\mathrm{PH}}+\mathrm{ex}_{\mathrm{CH}}+\mathrm{ex}_{\mathrm{KE}}+\mathrm{ex}_{\mathrm{PT}}
\end{aligned}
$$

where $\mathrm{ex}_{\mathrm{PH}}$, $\mathrm{ex}_{\mathrm{CH}}, \mathrm{ex}_{\mathrm{KE}}$, and $\mathrm{ex}_{\mathrm{PT}}$ denote physical, chemical, kinetic, and potential exergies.

Apart from the common mass, energy, and exergy balance equations a series of other equations are solved to complete the thermodynamic modeling of this study. The emitted heat from the sun is evaluated as follows [34]:

$$
\dot{\mathrm{Q}}_{\mathrm{s}}=\mathrm{A} \times \mathrm{n} \times \mathrm{I}
$$

Here, A, n, and I represent the aperture area, number of mirrors, and irradiance, respectively. A portion of the heat from the sun is reflected by the mirrors while the remaining heat is wasted due to the optical efficiency of the mirrors [34]:

$$
\dot{\mathrm{Q}}_{\mathrm{h}}=\dot{\mathrm{Q}}_{\mathrm{s}} \times \eta_{\mathrm{h}}
$$

where $\eta_{\mathrm{h}}$ is the optical efficiency of the heliostat mirrors.

The rate of absorbed heat by the high-temperature molten salt is evaluated by calculating the heat losses associated with the receiver tower:

$$
\dot{\mathrm{Q}}_{\mathrm{a}}=\dot{\mathrm{Q}}_{\mathrm{h}}-\dot{\mathrm{Q}}_{\mathrm{l}, \text { total }}=\dot{\mathrm{Q}}_{\mathrm{h}}-\dot{\mathrm{Q}}_{\mathrm{l}, \text { cond }}-\dot{\mathrm{Q}}_{\mathrm{l}, \text { conv }}-\dot{\mathrm{Q}}_{\mathrm{l}, \mathrm{e}}-\dot{\mathrm{Q}}_{\mathrm{l}, \mathrm{r}}
$$

where $\dot{\mathrm{Q}}_{\mathrm{l} \text {,total }}, \dot{\mathrm{Q}}_{\mathrm{l} \text {,cond }}, \dot{\mathrm{Q}}_{\mathrm{l}, \text { conv }}, \dot{\mathrm{Q}}_{\mathrm{l}, \mathrm{e}}$, and $\dot{\mathrm{Q}}_{\mathrm{l}, \mathrm{r}}$ denote total heat loss of the receiver, conduction heat loss, convection heat loss, emission heat loss, and radiation heat loss, respectively.

Considering charging and discharging modes, each system component is studied using the first and second laws of thermodynamics.

The reaction that takes place in the combustion chamber is given by [17]:

$$
\begin{aligned}
\bar{\lambda} \mathrm{C}_{\mathrm{x}_{1}} \mathrm{H}_{\mathrm{y}_{1}}+\left(\mathrm{x}_{\mathrm{N}_{2}} \mathrm{~N}_{2}+\mathrm{x}_{\mathrm{O}_{2}} \mathrm{O}_{2}+\mathrm{x}_{\mathrm{Ar}} \mathrm{Ar}\right) & \\
& \rightarrow \mathrm{y}_{\mathrm{CO}_{2}} \mathrm{CO}_{2}+\mathrm{y}_{\mathrm{N}_{2}} \mathrm{~N}_{2}+\mathrm{y}_{\mathrm{O}_{2}} \mathrm{O}_{2}+\mathrm{y}_{\mathrm{H}_{2} \mathrm{O}} \mathrm{H}_{2} \mathrm{O}+\mathrm{y}_{\mathrm{Ar}} \mathrm{Ar}
\end{aligned}
$$

Energy balance on the combustion chamber is expressed as follows:

$$
\dot{\mathrm{n}}_{\text {Air }} \overline{\mathrm{h}}_{\text {Air }}+\dot{\mathrm{n}}_{\text {Fuel }} L H V_{\text {Fuel }} \mathrm{M}_{\text {Fuel }}=\dot{\mathrm{n}}_{\text {Product }} \overline{\mathrm{h}}_{\text {Product }}+\left(1-\eta_{\text {CC }}\right) \mathrm{LHV}_{\text {Fuel }} \mathrm{M}_{\text {Fuel }}
$$

where LHV Fuel represents a lower heating value of the fuel.

The TEG power output is calculated using the following equations [35]:

$$
\begin{aligned}
& \dot{\mathrm{W}}_{\mathrm{TEG}}=\eta_{\mathrm{TEG}} / \dot{\mathrm{Q}}_{\mathrm{TEG}} \\
& \eta_{\mathrm{TEG}}=\eta_{\text {Carnot }} \times \frac{\sqrt{1+\mathrm{Z}_{\mathrm{M}}-1}}{\sqrt{1+\mathrm{Z}_{\mathrm{M}}+\frac{\mathrm{T}_{\mathrm{L}}}{\mathrm{T}_{\mathrm{H}}}}}
\end{aligned}
$$




$$
\begin{aligned}
& \mathrm{T}_{\mathrm{M}}=\frac{1}{2} \times\left(\mathrm{T}_{\mathrm{L}}+\mathrm{T}_{\mathrm{H}}\right) \\
& \dot{\mathrm{Q}}_{\mathrm{TEG}}=\dot{\mathrm{m}}_{15}\left(\mathrm{~h}_{16}-\mathrm{h}_{15}\right)
\end{aligned}
$$

where $\eta_{\text {Carnot }}, Z_{M}, T_{L}$, and $T_{H}$ are the Carnot efficiency, figure of merit, temperature of cold plate, and temperature of hot plate of the TEG unit.

The power consumed during charging, and also, the power produced during discharging mode are evaluated as follows:

$$
\begin{aligned}
& \dot{\mathrm{W}}_{\text {charge }}=\dot{\mathrm{W}}_{\mathrm{AC}, 1}+\dot{\mathrm{W}}_{\mathrm{AC}, 2} \\
& \dot{\mathrm{W}}_{\text {discharge }}=\dot{\mathrm{W}}_{\mathrm{GT}, 1}+\dot{\mathrm{W}}_{\mathrm{GT}, 2}+\dot{\mathrm{W}}_{\mathrm{TEG}}+\dot{\mathrm{W}}_{\mathrm{LAPC}, \mathrm{t}}+\dot{\mathrm{W}}_{\mathrm{NG}, \mathrm{t}}-\dot{\mathrm{W}}_{\mathrm{LAPC}, \mathrm{p}}-\dot{\mathrm{W}}_{\mathrm{LNG}, \mathrm{p}}
\end{aligned}
$$

where subscript AC, GT, TEG, NG, LAPC, t, and p denote air compressor, gas turbine, thermoelectric generator, natural gas, liquefied air power cycle, turbine, and pump, respectively.

Cooling load produced during discharge is given as follows:

$$
\mathrm{Q}_{\mathrm{C}}=\dot{\mathrm{m}}_{45} \times\left(\mathrm{h}_{45}-\mathrm{h}_{44}\right)
$$

Energy and exergy efficiencies of the integrated system are presented as follows:

$$
\begin{aligned}
& \eta_{\mathrm{I}}=\frac{\left(\dot{\mathrm{W}}_{\text {discharge }} \times \mathrm{t}_{\mathrm{d}}\right)+\left(\mathrm{Q}_{\mathrm{C}} \times \mathrm{t}_{\mathrm{d}}\right)}{\left(\dot{\mathrm{W}}_{\text {charge }} \times \mathrm{t}_{\mathrm{c}}\right)+\left(\dot{\mathrm{m}}_{38} \times\left(\mathrm{h}_{43}-\mathrm{h}_{38}\right)\right) \times \mathrm{t}_{\mathrm{d}}+\left(\dot{\mathrm{Q}}_{\mathrm{s}} \times \mathrm{t}_{\mathrm{d}}\right)+\left(\dot{\mathrm{m}}_{10} \times \mathrm{LHV} \times \mathrm{t}_{\mathrm{d}}\right)} \\
& \eta_{\mathrm{II}} \\
& =1-\frac{\left(\dot{\mathrm{Ex}}_{\mathrm{d}, \text { total,charge }} \times \mathrm{t}_{\mathrm{c}}\right)+\left(\dot{E}_{\mathrm{d}, \text { total, discharge }} \times \mathrm{t}_{\mathrm{d}}\right)}{\left(\dot{\mathrm{W}}_{\text {charge }} \times \mathrm{t}_{\mathrm{c}}\right)+\left(\dot{\mathrm{m}}_{38} \times\left(\mathrm{ex}_{\mathrm{f}, 38}-\mathrm{ex}_{\mathrm{f}, 43}\right)\right) \times \mathrm{t}_{\mathrm{d}}+\dot{\mathrm{Ex}}_{\mathrm{s}} \times \mathrm{t}_{\mathrm{d}}+\dot{\mathrm{m}}_{10} \times \mathrm{ex}_{\mathrm{f}, 10} \times \mathrm{t}_{\mathrm{d}}}
\end{aligned}
$$

Normalized carbon dioxide emission is determined by:

$$
\varepsilon=\frac{\dot{\mathrm{m}}_{\mathrm{CO}_{2}}}{\dot{\mathrm{W}}_{\text {net }}}
$$

Here, $\dot{\mathrm{m}}_{\mathrm{CO}_{2}}$ is the mass flow rate of carbon dioxide determined from the following equation:

$$
\dot{\mathrm{m}}_{\mathrm{CO}_{2}}=\frac{\bar{\lambda} \times \dot{\mathrm{m}}_{9} \times \mathrm{M}_{\mathrm{CO}_{2}}}{\mathrm{M}_{\mathrm{air}}}
$$

where $\mathrm{M}_{\mathrm{CO}_{2}}$ and $\mathrm{M}_{\text {air }}$ denote the molar mass of carbon dioxide and air, respectively. Also, $\bar{\lambda}$ is the ratio of fuel to air on a molar basis [17]:

$$
\bar{\lambda}=\frac{\mathrm{n}_{\text {Fuel }}}{\mathrm{n}_{\text {Air }}}
$$


Major input parameters are presented in Table 2 [23,34-36].

Table 2 Input parameters for the base case of this study.

\begin{tabular}{ll}
\hline Parameter & Value \\
\hline Mass flow rate of air entering the system $(\mathrm{kg} / \mathrm{s})$ & 100 \\
Charging time $(\mathrm{h})$ & 8 \\
Discharging time $(\mathrm{h})$ & 8 \\
Pressure ratio of each compression/expansion stage & 6 \\
Pinch point $\left({ }^{\circ} \mathrm{C}\right)$ & 10 \\
Area of a single mirror $\left(\mathrm{m}^{2}\right)$ & 100 \\
Number of mirrors & 400 \\
Optical efficiency $(\%)$ & 75 \\
Wind velocity $(\mathrm{m} / \mathrm{s})$ & 5 \\
Molten salt type & $(\mathrm{LiNaK})_{2} \mathrm{CO}_{3}$ \\
Inlet temperature of molten salt $\left({ }^{\circ} \mathrm{C}\right)$ & 400 \\
Outlet temperature of molten salt $\left({ }^{\circ} \mathrm{C}\right)$ & 700 \\
Outlet temperature of combustor $\left({ }^{\circ} \mathrm{C}\right)$ & 1100 \\
TEG figure of merit & 0.8 \\
Liquefied air power cycle turbine inlet pressure $(\mathrm{kPa})$ & 15000 \\
Pressure of LNG tank $(\mathrm{kPa})$ & 130 \\
Pressure of LNG pump outlet $(\mathrm{kPa})$ & 10000 \\
Temperature of liquefied air power cycle condenser $\left({ }^{\circ} \mathrm{C}\right)$ & $\mathrm{T}_{39}$ \\
& + pinch point \\
NG turbine outlet pressure $(\mathrm{kPa})$ & 7000 \\
Isentropic efficiency of air compressors $(\%)$ & 0.8 \\
Isentropic efficiency of gas turbines $(\%)$ & 0.86 \\
Isentropic efficiency of liquefied air power cycle and NG turbines $(\%)$ & 0.8 \\
Isentropic efficiency of liquefied air power cycle and LNG pumps $(\%)$ & 0.75 \\
\hline
\end{tabular}

Energy and exergy balance equations for each component present in the proposed energy storage system are presented in Table 3.

Table 3 Energy and exergy balance equations for each component of the integrated system

\begin{tabular}{|c|c|c|}
\hline Component & Energy balance equation & Exergy balance equation \\
\hline Air compressor I & $\mathrm{W}_{\mathrm{AC}, 1}=\left(\dot{\mathrm{m}}_{2} \mathrm{~h}_{2}-\dot{\mathrm{m}}_{1} \mathrm{~h}_{1}\right) \times \mathrm{t}_{\mathrm{c}}$ & $\mathrm{Ex}_{\mathrm{D}, \mathrm{AC}, 1}=\left(\dot{\mathrm{m}}_{1} \mathrm{ex}_{1}-\dot{\mathrm{m}}_{2} \mathrm{ex}_{2}+\dot{\mathrm{W}}_{\mathrm{AC}, 1}\right) \times \mathrm{t}_{\mathrm{c}}$ \\
\hline Air compressor II & $\mathrm{W}_{\mathrm{AC}, 2}=\left(\dot{\mathrm{m}}_{4} \mathrm{~h}_{4}-\dot{\mathrm{m}}_{3} \mathrm{~h}_{3}\right) \times \mathrm{t}_{\mathrm{c}}$ & $E x_{D, A C, 2}=\left(\dot{m}_{3} \mathrm{ex}_{3}-\dot{\mathrm{m}}_{4} \mathrm{ex}_{4}+\dot{\mathrm{W}}_{\mathrm{AC}, 2}\right) \times \mathrm{t}_{\mathrm{c}}$ \\
\hline Intercooler & $\begin{aligned}\left(\dot{\mathrm{m}}_{2} \mathrm{~h}_{2}+\dot{\mathrm{m}}_{18} \mathrm{~h}_{18}\right) & \times \mathrm{t}_{\mathrm{c}} \\
& =\left(\dot{\mathrm{m}}_{3} \mathrm{~h}_{3}\right. \\
& \left.+\dot{\mathrm{m}}_{20} \mathrm{~h}_{20}\right) \times \mathrm{t}_{\mathrm{c}}\end{aligned}$ & $\begin{aligned} \mathrm{Ex}_{\mathrm{D}, \mathrm{IC}}=\left(\dot{\mathrm{m}}_{2} \mathrm{ex}_{2}\right. & +\dot{\mathrm{m}}_{18} \mathrm{ex}_{18}-\dot{\mathrm{m}}_{3} \mathrm{ex}_{3} \\
& \left.-\dot{\mathrm{m}}_{20} \mathrm{ex}_{20}\right) \times \mathrm{t}_{\mathrm{c}}\end{aligned}$ \\
\hline Aftercooler & $\begin{aligned}\left(\dot{\mathrm{m}}_{4} \mathrm{~h}_{4}+\dot{\mathrm{m}}_{19} \mathrm{~h}_{19}\right) & \times \mathrm{t}_{\mathrm{c}} \\
& =\left(\dot{\mathrm{m}}_{5} \mathrm{~h}_{5}\right. \\
& \left.+\dot{\mathrm{m}}_{21} \mathrm{~h}_{21}\right) \times \mathrm{t}_{\mathrm{c}}\end{aligned}$ & $\begin{aligned} \mathrm{Ex}_{\mathrm{D}, \mathrm{AC}}=\left(\dot{\mathrm{m}}_{4} \mathrm{ex}_{4}\right. & +\dot{\mathrm{m}}_{19} \mathrm{ex}_{19}-\dot{\mathrm{m}}_{5} \mathrm{ex}_{5} \\
& \left.-\dot{\mathrm{m}}_{21} \mathrm{ex}_{21}\right) \times \mathrm{t}_{\mathrm{c}}\end{aligned}$ \\
\hline Heater I & $\begin{aligned}\left(\dot{\mathrm{m}}_{6} \mathrm{~h}_{6}+\dot{\mathrm{m}}_{23} \mathrm{~h}_{23}\right) & \times \mathrm{t}_{\mathrm{d}} \\
& =\left(\dot{\mathrm{m}}_{7} \mathrm{~h}_{7}\right. \\
& \left.+\dot{\mathrm{m}}_{24} \mathrm{~h}_{24}\right) \times \mathrm{t}_{\mathrm{d}}\end{aligned}$ & $\begin{array}{c}\mathrm{Ex}_{\mathrm{D}, \mathrm{H}, 1}=\left(\dot{\mathrm{m}}_{6} \mathrm{ex}_{6}+\dot{\mathrm{m}}_{23} \mathrm{ex}_{23}-\dot{\mathrm{m}}_{7} \mathrm{ex}_{7}\right. \\
\left.-\dot{\mathrm{m}}_{24} \mathrm{ex}_{24}\right) \times \mathrm{t}_{\mathrm{d}}\end{array}$ \\
\hline
\end{tabular}




\begin{tabular}{|c|c|c|}
\hline Heater II & $\begin{aligned}\left(\dot{\mathrm{m}}_{7} \mathrm{~h}_{7}+\dot{\mathrm{m}}_{30} \mathrm{~h}_{30}\right) & \times \mathrm{t}_{\mathrm{d}} \\
& =\left(\dot{\mathrm{m}}_{8} \mathrm{~h}_{8}\right. \\
& \left.+\dot{\mathrm{m}}_{31} \mathrm{~h}_{31}\right) \times \mathrm{t}_{\mathrm{d}}\end{aligned}$ & $\begin{array}{c}\mathrm{Ex}_{\mathrm{D}, \mathrm{H}, 2}=\left(\dot{\mathrm{m}}_{7} \mathrm{ex}_{7}+\dot{\mathrm{m}}_{30} \mathrm{ex}_{30}-\dot{\mathrm{m}}_{8} \mathrm{ex}_{8}\right. \\
\left.-\dot{\mathrm{m}}_{31} \mathrm{ex}_{31}\right) \times \mathrm{t}_{\mathrm{d}}\end{array}$ \\
\hline Gas turbine I & $\mathrm{W}_{\mathrm{GT}, 1}=\left(\dot{\mathrm{m}}_{8} \mathrm{~h}_{8}-\dot{\mathrm{m}}_{9} \mathrm{~h}_{9}\right) \times \mathrm{t}_{\mathrm{d}}$ & $\mathrm{Ex}_{\mathrm{D}, \mathrm{GT}, 1}=\left(\dot{\mathrm{m}}_{8} \mathrm{ex}_{8}-\dot{\mathrm{m}}_{9} \mathrm{ex}_{9}-\dot{\mathrm{W}}_{\mathrm{GT}, 1}\right) \times \mathrm{t}_{\mathrm{d}}$ \\
\hline Gas turbine II & $\mathrm{W}_{\mathrm{GT}, 2}=\left(\dot{\mathrm{m}}_{11} \mathrm{~h}_{11}-\dot{\mathrm{m}}_{12} \mathrm{~h}_{12}\right) \times \mathrm{t}_{\mathrm{d}}$ & $\mathrm{Ex}_{\mathrm{D}, \mathrm{GT}, 2}=\left(\dot{\mathrm{m}}_{1} \mathrm{ex}_{1}-\dot{\mathrm{m}}_{2} \mathrm{ex}_{2}-\dot{\mathrm{W}}_{\mathrm{GT}, 2}\right) \times \mathrm{t}_{\mathrm{d}}$ \\
\hline $\begin{array}{l}\text { Combustion } \\
\text { chamber }\end{array}$ & $\begin{aligned}\left(\dot{\mathrm{m}}_{9} \mathrm{~h}_{9}+\dot{\mathrm{m}}_{10} \mathrm{LHV}_{\text {Fuel }}\right) \times \mathrm{t}_{\mathrm{d}} & \\
& =\left(\dot{\mathrm{m}}_{11} \mathrm{~h}_{11}\right. \\
& \left.+\left(1-\eta_{\mathrm{CC}}\right) \mathrm{LHV}_{\text {Fuel }}\right) \\
& \times \mathrm{t}_{\mathrm{d}}\end{aligned}$ & $\begin{aligned} \mathrm{Ex}_{\mathrm{D}, \mathrm{CC}}=\left(\dot{\mathrm{m}}_{9} \mathrm{ex}_{9}\right. & +\dot{\mathrm{m}}_{10} \mathrm{ex}_{10} \\
& \left.-\dot{\mathrm{m}}_{11} \mathrm{ex}_{11}\right) \times \mathrm{t}_{\mathrm{d}}\end{aligned}$ \\
\hline Heater III & $\begin{aligned}\left(\dot{\mathrm{m}}_{12} \mathrm{~h}_{12}+\dot{\mathrm{m}}_{34} \mathrm{~h}_{34}\right) \times \mathrm{t}_{\mathrm{d}} & \\
& =\left(\dot{\mathrm{m}}_{13} \mathrm{~h}_{13}\right. \\
& \left.+\dot{\mathrm{m}}_{35} \mathrm{~h}_{35}\right) \times \mathrm{t}_{\mathrm{d}}\end{aligned}$ & $\begin{array}{c}\mathrm{Ex}_{\mathrm{D}, \mathrm{H}, 3}=\left(\dot{\mathrm{m}}_{12} \mathrm{ex}_{12}+\dot{\mathrm{m}}_{34} \mathrm{ex}_{34}-\dot{\mathrm{m}}_{13} \mathrm{ex}_{13}\right. \\
\left.-\dot{\mathrm{m}}_{35} \mathrm{ex}_{35}\right) \times \mathrm{t}_{\mathrm{d}}\end{array}$ \\
\hline LAPC turbine & $\mathrm{W}_{\mathrm{LAPC}, \mathrm{t}}=\left(\dot{\mathrm{m}}_{35} \mathrm{~h}_{35}-\dot{\mathrm{m}}_{36} \mathrm{~h}_{36}\right) \times \mathrm{t}_{\mathrm{d}}$ & $\begin{aligned} \mathrm{Ex}_{\mathrm{D}, \mathrm{LAPC}, \mathrm{t}}=\left(\dot{\mathrm{m}}_{35} \mathrm{ex}_{35}-\dot{\mathrm{m}}_{36} \mathrm{ex}_{36}\right. & \left.-\dot{\mathrm{W}}_{\mathrm{LAPC}, \mathrm{t}}\right) \times \mathrm{t}_{\mathrm{d}}\end{aligned}$ \\
\hline Recuperator & $\begin{aligned}\left(\dot{\mathrm{m}}_{33} \mathrm{~h}_{33}+\dot{\mathrm{m}}_{36} \mathrm{~h}_{36}\right) \times \mathrm{t}_{\mathrm{d}} & \\
& =\left(\dot{\mathrm{m}}_{34} \mathrm{~h}_{34}\right. \\
& \left.+\dot{\mathrm{m}}_{37} \mathrm{~h}_{37}\right) \times \mathrm{t}_{\mathrm{d}}\end{aligned}$ & $\begin{array}{c}\mathrm{Ex}_{\mathrm{D}, \mathrm{REC}}=\left(\dot{\mathrm{m}}_{33} \mathrm{ex}_{33}+\dot{\mathrm{m}}_{36} \mathrm{ex}_{36}-\dot{\mathrm{m}}_{34} \mathrm{ex}_{34}\right. \\
\left.-\dot{\mathrm{m}}_{37} \mathrm{ex}_{37}\right) \times \mathrm{t}_{\mathrm{d}}\end{array}$ \\
\hline Condenser & $\begin{aligned}\left(\dot{\mathrm{m}}_{37} \mathrm{~h}_{37}+\dot{\mathrm{m}}_{39} \mathrm{~h}_{39}\right) \times \mathrm{t}_{\mathrm{d}} & \\
& =\left(\dot{\mathrm{m}}_{32} \mathrm{~h}_{32}\right. \\
& \left.+\dot{\mathrm{m}}_{40} \mathrm{~h}_{40}\right) \times \mathrm{t}_{\mathrm{d}}\end{aligned}$ & $\begin{array}{c}\mathrm{Ex}_{\mathrm{D}, \mathrm{C}}=\left(\dot{\mathrm{m}}_{37} \mathrm{ex}_{37}+\dot{\mathrm{m}}_{39} \mathrm{ex}_{39}-\dot{\mathrm{m}}_{32} \mathrm{ex}_{32}\right. \\
\left.-\dot{\mathrm{m}}_{40} \mathrm{ex}_{40}\right) \times \mathrm{t}_{\mathrm{d}}\end{array}$ \\
\hline LAPC pump & $\mathrm{W}_{\mathrm{LAPC}, \mathrm{p}}=\left(\dot{\mathrm{m}}_{33} \mathrm{~h}_{33}-\dot{\mathrm{m}}_{32} \mathrm{~h}_{32}\right) \times \mathrm{t}_{\mathrm{d}}$ & $\begin{array}{r}\mathrm{Ex}_{\mathrm{D}, \mathrm{LAPC}, \mathrm{p}}=\left(\dot{\mathrm{m}}_{32} \mathrm{ex}_{32}-\dot{\mathrm{m}}_{33} \mathrm{ex}_{33}\right. \\
\left.+\dot{\mathrm{W}}_{\mathrm{LAPC}, \mathrm{p}}\right) \times \mathrm{t}_{\mathrm{d}}\end{array}$ \\
\hline LNG pump & $\mathrm{W}_{\mathrm{LNG}, \mathrm{p}}=\left(\dot{\mathrm{m}}_{39} \mathrm{~h}_{39}-\dot{\mathrm{m}}_{38} \mathrm{~h}_{38}\right) \times \mathrm{t}_{\mathrm{d}}$ & $\begin{aligned} \mathrm{Ex}_{\mathrm{D}, \mathrm{LNG}, \mathrm{p}}=\left(\dot{\mathrm{m}}_{38} \mathrm{ex}_{38}-\dot{\mathrm{m}}_{39} \mathrm{ex}_{39}\right. & \\
& \left.+\dot{\mathrm{W}}_{\mathrm{LNG}, \mathrm{p}}\right) \times \mathrm{t}_{\mathrm{d}}\end{aligned}$ \\
\hline Heater IV & $\begin{aligned}\left(\dot{\mathrm{m}}_{26} \mathrm{~h}_{26}+\dot{\mathrm{m}}_{40} \mathrm{~h}_{40}\right) \times \mathrm{t}_{\mathrm{d}} & \\
& =\left(\dot{\mathrm{m}}_{27} \mathrm{~h}_{27}\right. \\
& \left.+\dot{\mathrm{m}}_{41} \mathrm{~h}_{41}\right) \times \mathrm{t}_{\mathrm{d}}\end{aligned}$ & $\begin{array}{c}\mathrm{Ex}_{\mathrm{D}, \mathrm{H}, 4}=\left(\dot{\mathrm{m}}_{26} \mathrm{ex}_{26}+\dot{\mathrm{m}}_{40} \mathrm{ex}_{40}-\dot{\mathrm{m}}_{27} \mathrm{ex}_{27}\right. \\
\left.-\dot{\mathrm{m}}_{41} \mathrm{ex}_{41}\right) \times \mathrm{t}_{\mathrm{d}}\end{array}$ \\
\hline Heater V & $\begin{aligned}\left(\dot{\mathrm{m}}_{41} \mathrm{~h}_{41}+\dot{\mathrm{m}}_{44} \mathrm{~h}_{44}\right) \times \mathrm{t}_{\mathrm{d}} & \\
& =\left(\dot{\mathrm{m}}_{42} \mathrm{~h}_{42}\right. \\
& \left.+\dot{\mathrm{m}}_{45} \mathrm{~h}_{45}\right) \times \mathrm{t}_{\mathrm{d}}\end{aligned}$ & $\begin{array}{c}\mathrm{Ex}_{\mathrm{D}, \mathrm{H}, 5}=\left(\dot{\mathrm{m}}_{41} \mathrm{ex}_{41}+\dot{\mathrm{m}}_{44} \mathrm{ex}_{44}-\dot{\mathrm{m}}_{42} \mathrm{ex}_{42}\right. \\
\left.-\dot{\mathrm{m}}_{45} \mathrm{ex}_{45}\right) \times \mathrm{t}_{\mathrm{d}}\end{array}$ \\
\hline Natural gas turbine & $\mathrm{W}_{\mathrm{NG}, \mathrm{t}}=\left(\dot{\mathrm{m}}_{42} \mathrm{~h}_{42}-\dot{\mathrm{m}}_{43} \mathrm{~h}_{43}\right) \times \mathrm{t}_{\mathrm{d}}$ & $\begin{aligned} \mathrm{Ex}_{\mathrm{D}, \mathrm{NG}, \mathrm{t}}=\left(\dot{\mathrm{m}}_{42} \mathrm{ex}_{42}-\dot{\mathrm{m}}_{43} \mathrm{ex}_{43}\right. \\
\left.-\dot{\mathrm{W}}_{\mathrm{NG}, \mathrm{t}}\right) \times \mathrm{t}_{\mathrm{d}}\end{aligned}$ \\
\hline Heliostat mirrors & $\mathrm{Q}_{\mathrm{s}}=\left(\dot{\mathrm{Q}}_{\mathrm{h}}+\dot{\mathrm{Q}}_{\mathrm{l}, \mathrm{opt}}\right) \times \mathrm{t}_{\mathrm{d}}$ & $\mathrm{Ex}_{\mathrm{D}, \mathrm{HM}}=\left(\dot{E x_{s}}-\dot{E x_{h}}\right) \times t_{d}$ \\
\hline Solar tower & $\begin{array}{c}\mathrm{Q}_{\mathrm{h}}=\left(\dot{\mathrm{Q}}_{\mathrm{a}}+\dot{\mathrm{Q}}_{\mathrm{l}, \text { cond }}+\dot{\mathrm{Q}}_{\mathrm{l}, \text { conv }}+\dot{\mathrm{Q}}_{\mathrm{l}, \mathrm{e}}\right. \\
\left.+\dot{\mathrm{Q}}_{\mathrm{l}, \mathrm{r}}\right) \times \mathrm{t}_{\mathrm{d}}\end{array}$ & $\mathrm{Ex}_{\mathrm{D}, \mathrm{ST}}=\left(\dot{\mathrm{Ex}}_{\mathrm{h}}+\dot{\mathrm{m}}_{28} \mathrm{ex}_{28}-\dot{\mathrm{m}}_{29} \mathrm{ex}_{29}\right) \times \mathrm{t}_{\mathrm{d}}$ \\
\hline TEG & $\begin{aligned} \mathrm{W}_{\text {TEG }}=\left(\dot{\mathrm{m}}_{13} \mathrm{~h}_{13}\right. & +\dot{\mathrm{m}}_{15} \mathrm{~h}_{15}-\dot{\mathrm{m}}_{14} \mathrm{~h}_{14} \\
& \left.-\dot{\mathrm{m}}_{16} \mathrm{~h}_{16}\right) \times \mathrm{t}_{\mathrm{d}}\end{aligned}$ & $\begin{array}{r}\mathrm{Ex}_{\mathrm{D}, \mathrm{TEG}}=\left(\dot{\mathrm{m}}_{13} \mathrm{ex}_{13}+\dot{\mathrm{m}}_{15} \mathrm{ex}_{15}-\dot{\mathrm{m}}_{14} \mathrm{ex}_{14}\right. \\
\left.-\dot{\mathrm{m}}_{16} \mathrm{ex}_{16}-\dot{\mathrm{W}}_{\mathrm{TEG}}\right) \times \mathrm{t}_{\mathrm{d}}\end{array}$ \\
\hline
\end{tabular}

To perform the economic analysis, total revenue requirement method (TRR) is used. TRR for each year includes a return on investments, recovery of the capital, income tax, fuel costs, insurance and other taxes, and operation and maintenance costs [37]:

$$
\mathrm{TRR}_{\mathrm{j}}=\mathrm{ROI}_{\mathrm{j}, \mathrm{d}}+\mathrm{ROI}_{\mathrm{j}, \mathrm{ce}}+\mathrm{ROI}_{\mathrm{j}, \mathrm{ps}}+\mathrm{TCR}_{\mathrm{j}}+\mathrm{ITX}_{\mathrm{j}}+\mathrm{FC}_{\mathrm{j}}+\mathrm{OTXI}_{\mathrm{j}}+\mathrm{OMC}_{\mathrm{j}}
$$

where subscripts d, ce, and ps denote debt, common equity, and preffered stock, respectively. Levelized TRR is then evaluated by [37]: 


$$
\mathrm{TRR}_{\mathrm{L}}=\mathrm{CRF} \sum_{1}^{\mathrm{n}} \frac{\mathrm{TRR}_{\mathrm{j}}}{(1+\mathrm{i})^{\mathrm{j}}}
$$

where CRF is the capital recovery factor, $\mathrm{n}$ is the lifetime of plant, and $\mathrm{i}$ is the interest rate [37]. Levelized cost of electricity is obtained as follows [37]:

$$
\mathrm{LCOE}=\frac{\mathrm{TRR}_{\mathrm{L}}-\mathrm{BPV}}{\mathrm{MPQ}}
$$

Here, MPQ and BPV are main product quantity and byproduct value, respectively [37]. The ssumptions used for the economic analysis are listed in Table 4 [37].

Table 4 Economic inputs and assumptions used for economic analysis.

\begin{tabular}{ll}
\hline Parameter & Value \\
\hline Lifetime of plant (years) & 30 \\
Interest rate (\%) & 6 \\
ROR of debt $(\%)$ & 15 \\
ROR common equity (\%) & 11.7 \\
ROR preferred stock (\%) & 10 \\
Debt fraction of total investment cost $(\%)$ & 35 \\
Common equity fraction of total investment cost $(\%)$ & 15 \\
Preferred stock of total investment cost $(\%)$ & 50 \\
O\&M factor $(\%)$ & 2 \\
Tax life of plant (years) & 15 \\
Rate of income tax (\%) & 30 \\
Other taxes and insurance factor $(\%)$ & 2 \\
Cost of LNG (\$/GJ) & 5.66 \\
Cost of natural gas (\$/GJ) & 3.15 \\
Reference economic year & 2020 \\
\hline
\end{tabular}

For evaluation of total capital investment, it is necessary to calculate the purchase equipment costs (PEC). Cost functions listed in Table 5 are used to evaluate the total PEC of the plant [3742]. Total PEC of the plant is around $23 \%$ of the fixed capital investment, while installation of equipments and instrumentation account for $12 \%$ of the fixed capital investment. Piping, electrical, building, yard improvement, service facilities, land, engineering and supervision, expenses of construction, contractor's fee, contingencies, and working capital investment account for 7, 4, 8, $2,13,1,9,10,2,9$, and $17.5 \%$ of the fixed capital investment, respectively [37].

Table 5 Cost functions used for the estimation of total capital invesetment.

\begin{tabular}{ll}
\hline Component & Cost function \\
\hline Heliostat mirrors & $\mathrm{Z}=145 \mathrm{~N}_{\text {helio }} \mathrm{A}_{\text {helio }}$ \\
Solar receiver & $\mathrm{Z}=1.03 \times 10^{8}\left(\mathrm{~A}_{\text {rec }} / 1571\right)^{0.7}$ \\
Solar tower & $\mathrm{Z}=300 \times \exp \left(0.0113\left(\mathrm{H}_{\text {tower }}-\left(\mathrm{H}_{\text {rec }}-\mathrm{H}_{\text {helio }}\right) / 2\right)\right.$ \\
Storage tanks & $\mathrm{Z}=2.9232 \exp \left(11.662-0.6104\left(\ln (\mathrm{V})+0.04536(\ln (\mathrm{V}))^{2}\right)\right)$ \\
Molten salt & $\mathrm{Z}=1.2 \mathrm{~m}$
\end{tabular}




$\begin{array}{ll}\text { Air compressor } & \mathrm{Z}=\frac{71.1 \dot{\mathrm{m}}}{0.92-\eta} \times \mathrm{R}_{\mathrm{p}} \times \ln \left(\mathrm{R}_{\mathrm{p}}\right) \\ \text { Gas turbine } & \mathrm{Z}=\frac{479.34 \dot{\mathrm{m}}}{0.92-\eta} \times \ln \left(\mathrm{R}_{\mathrm{p}}\right) \times\left(1+\exp \left(0.036 \mathrm{~T}_{\mathrm{i}}-54.4\right)\right) \\ \text { Combustion chamber } & \mathrm{Z}=\frac{46.08 \dot{\mathrm{m}}_{\text {air }}}{0.995-\left(\mathrm{P}_{\text {outlet }} / \mathrm{P}_{\text {inlet }}\right)}\left(1+\exp \left(0.018 \mathrm{~T}_{\text {outlet }}-26.4\right)\right) \\ \text { Pump } & \mathrm{Z}=705.48(\dot{\mathrm{W}})^{0.7}\left(1+\frac{0.2}{1-\eta}\right) \\ \text { Heat exchangers } & \mathrm{Z}=2143(\mathrm{~A})^{0.514} \\ \text { Condenser } & \mathrm{Z}=1397\left(\mathrm{~A}^{0.89}\right) \\ \text { TEG } & \mathrm{Z}=1500(\dot{\mathrm{W}})\end{array}$

\section{Results and Discussion}

The results of the energy and exergy analyses of the integrated system proposed in this study are presented in Table 6. Using Eqs. 15 and 16, the energy and exergy efficiencies are found as $55.3 \%$ and $46.4 \%$, respectively. Additionally, it is observed that for producing $797.6 \mathrm{MWh}$ of electricity during discharge, 63.4 tons/day of fuel is required. The charging power required for the compressors is $356 \mathrm{MWh}$. The terms of Eqs. 12 and 13 are calculated by using the energy balance equations listed in Table 3. By applying the balance equations on the two air compressor, power required for the charging mode of the plant is calculated. Also, the cooling load produced during discharging mode is calculated by Eq. 14. Moreover, by using the exergy balance equations listed in Table 3, the overall exergy destruction of the plant is found to be 1,035.2 MWh. In the next section of results and discussion, detailed results of the exergy analysis will be presented.

After determining the unknown species coefficients of the combustion process (see Eq. 10) and the molar based fuel to air ratio (see Eq. 19), the mass of produced carbon dioxide is calculated using Eq. 18. Finally, by knowing the net power generation of the plant, the normalized carbon dioxide emissions are calculated as $210.1 \mathrm{~kg} \mathrm{CO}_{2} / \mathrm{MWh}$ (see Eq. 17). Thermodynamic properties including temperature, pressure, enthalpy, and entropy for the various streams of the system are presented in Table 7. As mentioned earlier, for evaluating the thermophysical properties, REFPROP 9 library is utilized in this study. Also, energy balance equations listed in Table 3 are used to find unknown properties such as mass flow rate, enthalpy, temperature, or pressure. For instance, the enthalpy and temperature of state 8 , are calculated from the energy balance equation for heater 2 .

Table 6 Thermodynamic modelling results for the proposed system.

\begin{tabular}{ll}
\hline Parameter & Value \\
\hline Energy efficiency (\%) & 55.3 \\
Exergy efficiency (\%) & 46.4 \\
Power consumption during charging (MWh) & 356 \\
Power generation during discharging (MWh) & 797.6 \\
Cooling power produced during discharging (MWh) & 423.2 \\
Overall exergy destruction (MWh) & $1,035.2$ \\
Consumed fuel (tons/day) & 63.4
\end{tabular}


Rate of consumed LNG (tons/day)

Required volume of the salt cavern $\left(\mathrm{m}^{3}\right)$

Total capital investment (Million dollars)

Levelized cost of electricity $(\phi / \mathrm{kWh})$

Normalized carbon dioxide emissions ( $\left.\mathrm{kg} \mathrm{CO}_{2} / \mathrm{MWh}\right)$
$5,539.5$

$100,188.3$

248.6

12.4

210.1

Table 7 Thermodynamic properties of various streams in the proposed solar-assisted CAES system.

\begin{tabular}{|c|c|c|c|c|c|c|}
\hline $\begin{array}{l}\text { Stream } \\
\text { number }\end{array}$ & Fluid & $\begin{array}{l}\text { State of } \\
\text { matter }\end{array}$ & $\begin{array}{l}\text { Pressure } \\
\text { (kPa) }\end{array}$ & $\begin{array}{l}\text { Temperature } \\
\left({ }^{\circ} \mathrm{C}\right)\end{array}$ & $\begin{array}{l}\text { Enthalpy } \\
\text { (kJ/kg) }\end{array}$ & $\begin{array}{l}\text { Entropy } \\
\text { (kJ/kg.K) }\end{array}$ \\
\hline 1 & Air & Gas & 101.3 & 25.2 & 298.4 & 6.9 \\
\hline 2 & Air & Gas & 506.5 & 240.3 & 517 & 6.9 \\
\hline 3 & Air & Gas & 506.5 & 35.2 & 307.7 & 6.4 \\
\hline 4 & Air & Gas & 2532.5 & 257.6 & 534.3 & 6.5 \\
\hline 5 & Air & Gas & 2532.5 & 35.2 & 303.4 & 6 \\
\hline 6 & Air & Gas & 2532.5 & 35.2 & 303.4 & 6 \\
\hline 7 & Air & Gas & 2532.5 & 227 & 502.4 & 6.5 \\
\hline 8 & Air & Gas & 2532.5 & 448.4 & 737.4 & 6.8 \\
\hline 9 & Air & Gas & 506.5 & 227.8 & 504 & 6.9 \\
\hline 10 & Methane & Gas & 101.3 & 25.2 & 910 & 6.7 \\
\hline 11 & Flue gas & Gas & 506.5 & 1100 & 1484.1 & 8 \\
\hline 12 & Flue gas & Gas & 101.3 & 714.6 & 1032.6 & 8.1 \\
\hline 13 & Flue gas & Gas & 101.3 & 368.3 & 651.1 & 7.6 \\
\hline 14 & Flue gas & Gas & 101.3 & 150 & 424.7 & 7.2 \\
\hline 15 & Water & Liquid & 101.3 & 25.2 & 104.9 & 0.4 \\
\hline 16 & Water & Liquid & 101.3 & 30.2 & 125.8 & 0.4 \\
\hline 17 & Therminol 66 & Liquid & 101.3 & 25.2 & 52.8 & 0.2 \\
\hline 18 & Therminol 66 & Liquid & 101.3 & 25.2 & 52.8 & 0.2 \\
\hline 19 & Therminol 66 & Liquid & 101.3 & 25.2 & 52.8 & 0.2 \\
\hline 20 & Therminol 66 & Liquid & 101.3 & 228.3 & 479.5 & 1.3 \\
\hline 21 & Therminol 66 & Liquid & 101.3 & 245.6 & 515.8 & 1.3 \\
\hline 22 & Therminol 66 & Liquid & 101.3 & 237 & 497.8 & 1.3 \\
\hline 23 & Therminol 66 & Liquid & 101.3 & 237 & 497.8 & 1.3 \\
\hline 24 & Therminol 66 & Liquid & 101.3 & 47.2 & 99 & 0.3 \\
\hline 25 & Therminol 66 & Liquid & 101.3 & 237 & 497.8 & 1.3 \\
\hline 26 & Therminol 66 & Liquid & 101.3 & 141.3 & 296.7 & 0.9 \\
\hline 27 & Therminol 66 & Liquid & 101.3 & 26.2 & 54.9 & 0.2 \\
\hline 28 & Carbonate salt & Liquid & 101.3 & 400 & 644 & 1.5 \\
\hline 29 & Carbonate salt & Liquid & 101.3 & 700 & 1127 & 2 \\
\hline 30 & Carbonate salt & Liquid & 101.3 & 700 & 1127 & 2 \\
\hline 31 & Carbonate salt & Liquid & 101.3 & 400 & 644 & 1.5 \\
\hline 32 & Air & Liquid & 3401 & -143.2 & -0.6 & 4.1 \\
\hline 33 & Air & Gas & 15000 & -119.6 & 30.7 & 4.2 \\
\hline 34 & Air & Gas & 15000 & 356.3 & 639.6 & 6.2 \\
\hline 35 & Air & Gas & 15000 & 704.6 & 1030.2 & 6.7 \\
\hline 36 & Air & Gas & 3401 & 455.5 & 745.4 & 6.8 \\
\hline 37 & Air & Gas & 3401 & -109.6 & 136.5 & 5.1 \\
\hline
\end{tabular}




\begin{tabular}{lllllll}
38 & Natural gas & Liquid & 130 & -158.2 & 10.9 & 0.1 \\
39 & Natural gas & Gas & 10000 & -153.2 & 42.4 & 0.2 \\
40 & Natural gas & Gas & 10000 & -121.6 & 153.7 & 1 \\
41 & Natural gas & Gas & 10000 & -75 & 348.3 & 2.1 \\
42 & Natural gas & Gas & 10000 & 15.2 & 778.8 & 3.9 \\
43 & Natural gas & Gas & 7000 & -7.9 & 745.5 & 4 \\
44 & R134a & Gas & 101.3 & 25.2 & 424.5 & 1.9 \\
45 & R134a & Liquid & 101.3 & -60 & 123.2 & 0.7 \\
\hline
\end{tabular}

The power consumption/generation of each system component is presented in Fig. 2. The first and second stages of the air compressor unit consume 174.9 and 181.3 MWh of electricity daily, respectively. The power produced by the first and second stages of the gas turbine are 186.7 and $369 \mathrm{MWh}$, respectively. The difference is due to the high difference in the inlet temperatures of gas entering the turbines. Inlet temperatures of the first and second stages are 448.4 and $1,100{ }^{\circ} \mathrm{C}$, respectively. Therefore, when the mass flow rate of gas remains constant, the high temperature of gas means higher enthalpy difference, and therefore, higher values of power is produced. The turbine of the liquefied air power cycle also produces 227.3 MWh of electricity which is quite significant, mainly due to the high heat addition and low heat rejection temperatures of this cycle. The power generated by the TEG for the waste heat recovery process is calculated as $52.8 \mathrm{MWh}$ daily. Moreover, the electric power consumed by the LNG and liquefied air power cycle pumps are calculated as 30.9 and $32.7 \mathrm{MWh}$, respectively. Also, the power produced in the natural gas expander is evaluated as $32.75 \mathrm{MWh}$ per day.

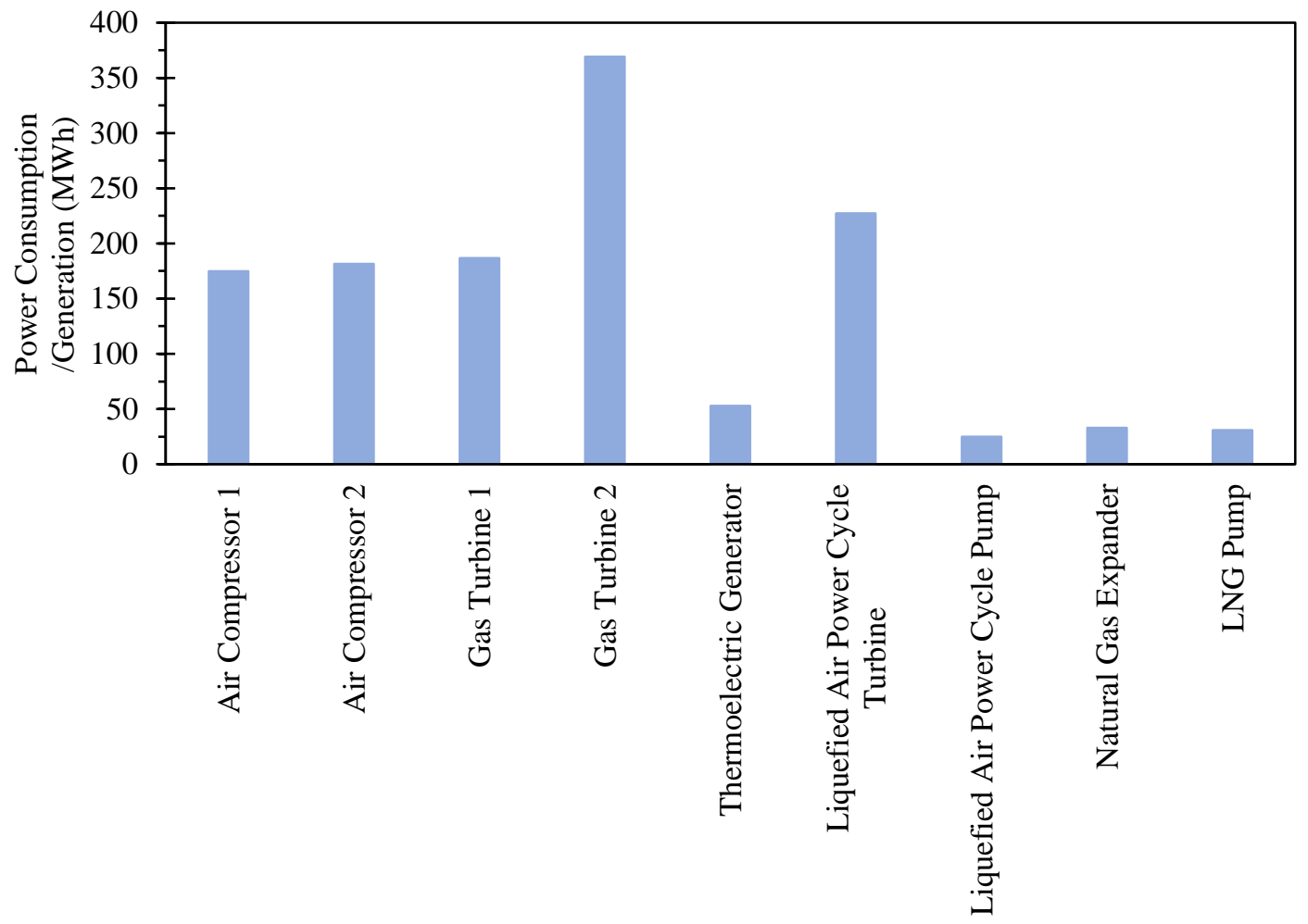


Figure 2 Power consumption/generation of various components in the integrated system.

Dynamic performance of the solar power tower in the integrated CAES system along the DNI of the selected location for a typical day is presented in Fig. 3. The solar irradiation starts from 6 A.M. as the sun rises and reach a maximum of $976.9 \mathrm{~W} / \mathrm{m}^{2}$ at 11 A.M. The maximum solar heat input to the heliostat mirrors is evaluated as $39.1 \mathrm{MW}$. Consider the heat losses associated with the heliostats and solar receiver, the net abosrbed heat by the molten salt is $23.74 \mathrm{MW}$. Therefore, the efficiency of the solar subsystem is $60.7 \%$ at 11 A.M.

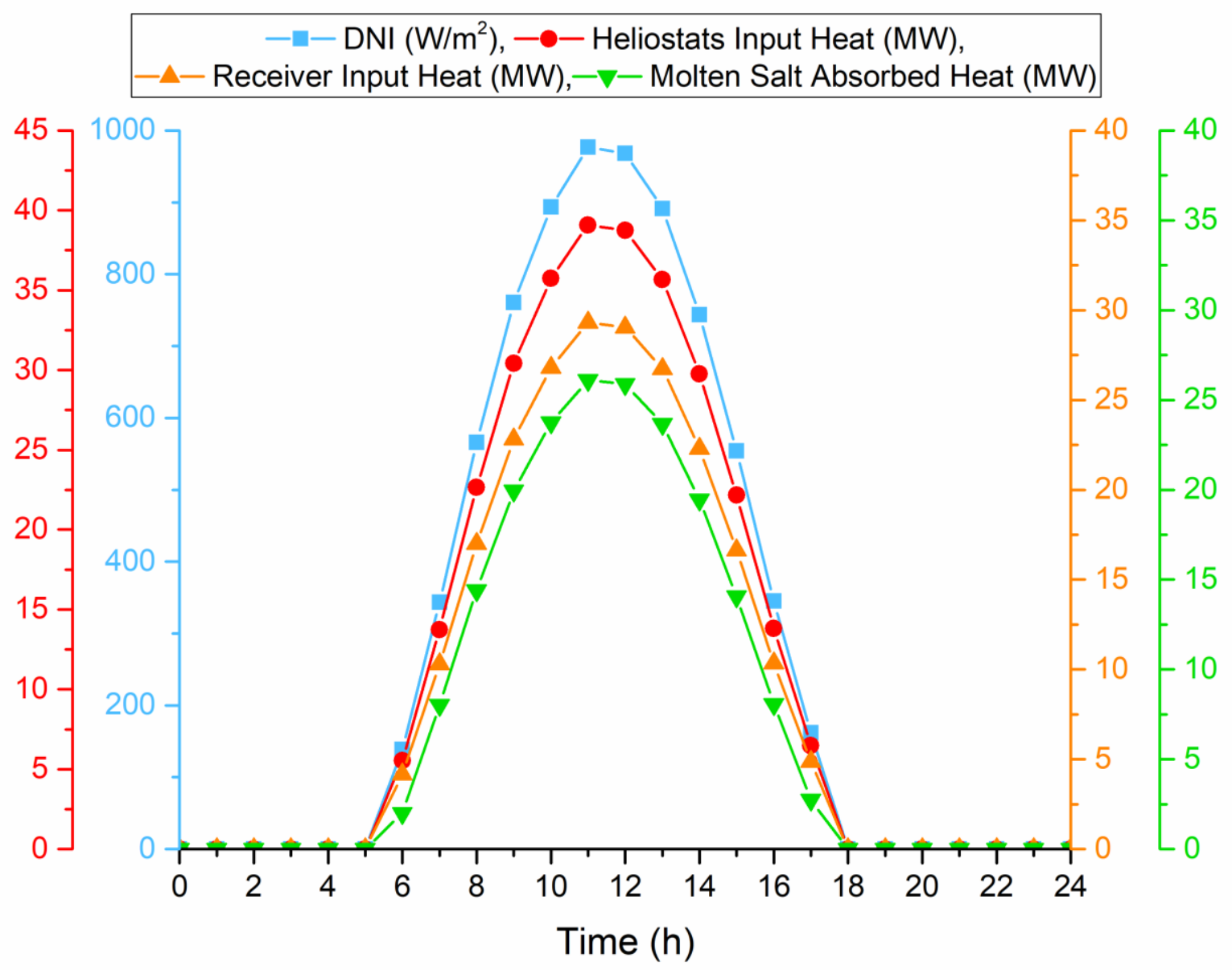

Figure 3 Dynamic performance of the solar power tower subsystem along the DNI of the selected location for a typical day in year.

Grid power storage and power generation of the energy storage system proposed in this study are shown in Fig. 4. From about 2 to 9 A.M., the demand profile of the selected location, which is the Bandar Abbas city located in southern Iran, is below the hourly average power demand. Therefore, the charging time of the plant for the grid power storage is set as 8 hours from 2 to 9 A.M. The power demand starts to rise steadily at around 7 A.M. and exceeds the average hourly 
demand from around 1 P.M., and reaches its peak at around 3 P.M. The electricity demand decreases significantly from around 9 P.M. Therefore, the discharging mode of the CAES plant is set from 1 P.M. to 9 P.M. to include the time period that the grid demand is high.

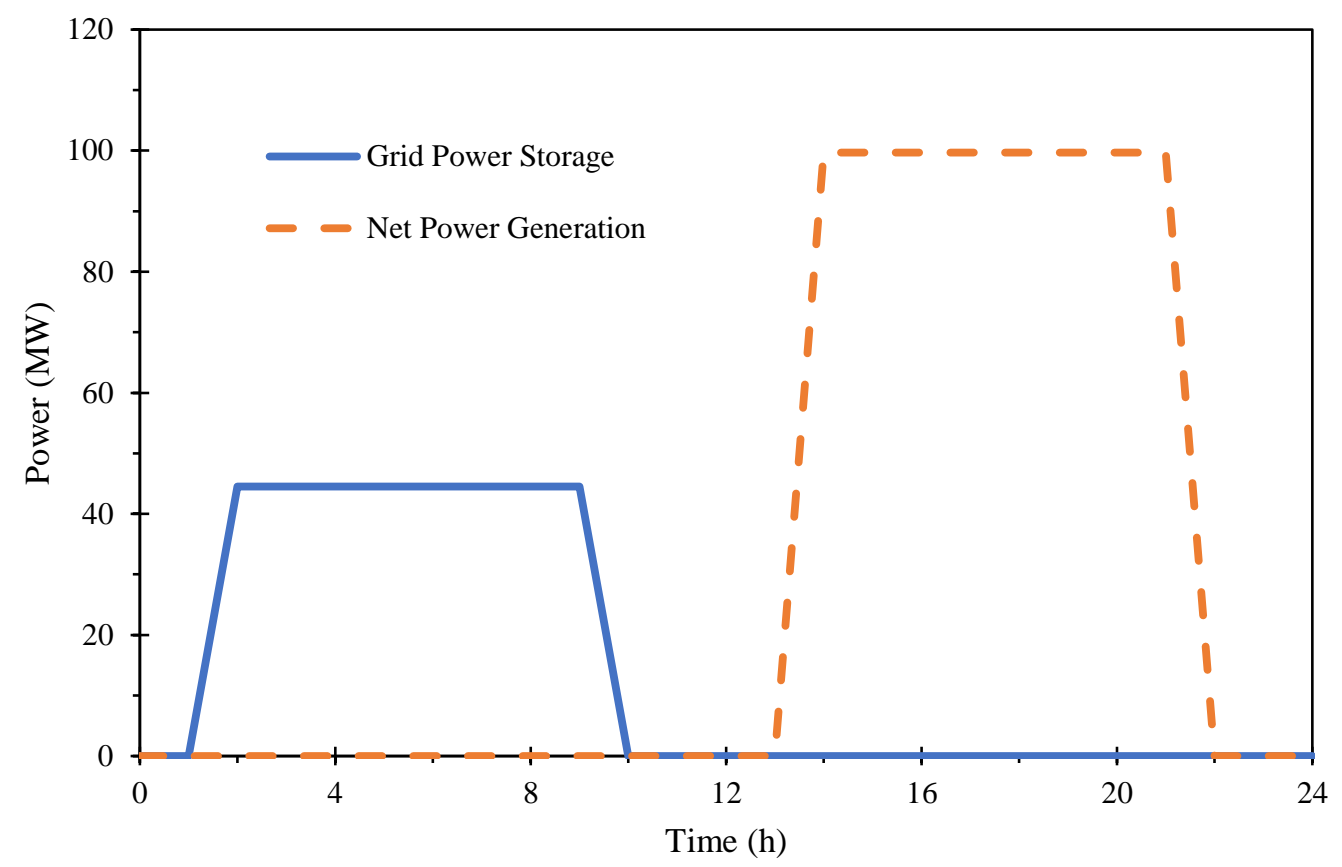

Figure 4 Grid power storage and power generation of the plant.

Exergy destruction of various components in the integrated system are presented in Fig. 5. As can be seen, the highest contributors to the overall exergy destruction of the system are the combustion chamber, heater 4 , solar tower, recuperator, and heliostats with a value of 370.9, 164.9, 89.8, 81.5, and 69.7 MWh, respectively. The high exergy destruction in the combustion chamber unit is due to the significant loss of available chemical exergy of the fuel during the combustion process. The significant exergy destruction rate of heater 4 is because of the high-temperature difference between the hot and cold streams of thermal oil and natural gas flowing through this heat exchanger. Similarly, high temperature difference between the hot and cold streams in the recuperator is the reason for its high exergy destruction. The exergy destruction of the solar tower unit is higher compared to heliostats because of the utilization of high-temperature salt. If a lower temperature salt is utilized, the exergy destruction of the solar tower unit will reduce. 


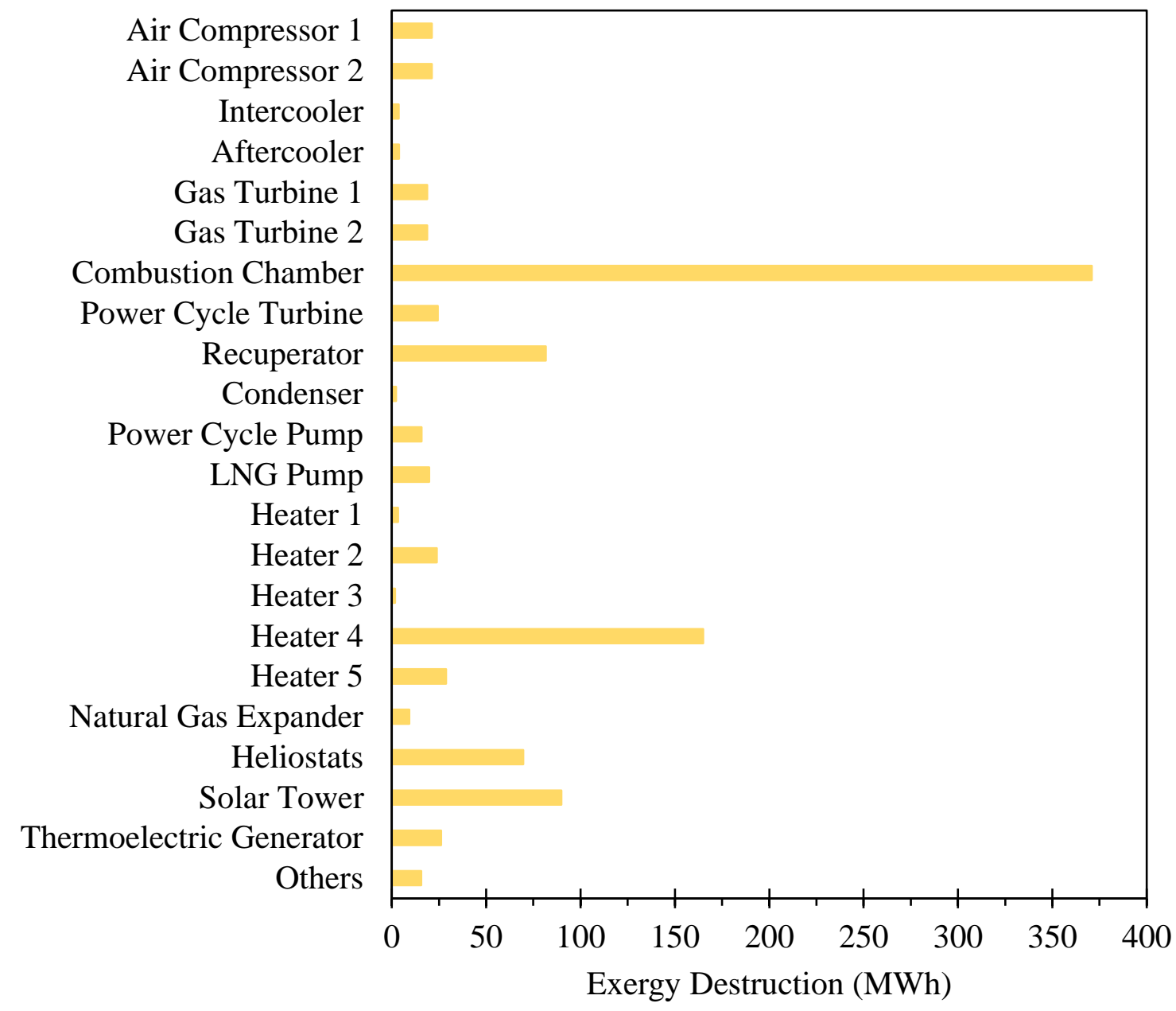

Figure 5 Exergy destruction of various components in the integrated system.

The effects of the gas turbines/compressors pressure ratios on the performance of the integrated system are presented in Fig. 6. By enhancing the pressure ratio of each stage of the compression/expansion process from 3 to 8 , the energy efficiency reduces from $56.5 \%$ to $53.6 \%$, while the daily exergy destruction increases from 1,013.1 to 1,094.2 MWh. As the pressure ratio increases, more heat is stored by the thermal oil and hence, the exergy destruction in heater 1 is increased. However, due to the higher temperature of the air at state 7, the exergy destruction of heater 2 reduces. On the other hand, the outlet temperature of the air at state 9 reduces as the pressure ratio increases. It will result in more fuel consumption to achieve the fixed gas turbine inlet temperature at state 11. An increase in fuel consumption causes an aggravation of the exergy destruction rate of the integrated system. 


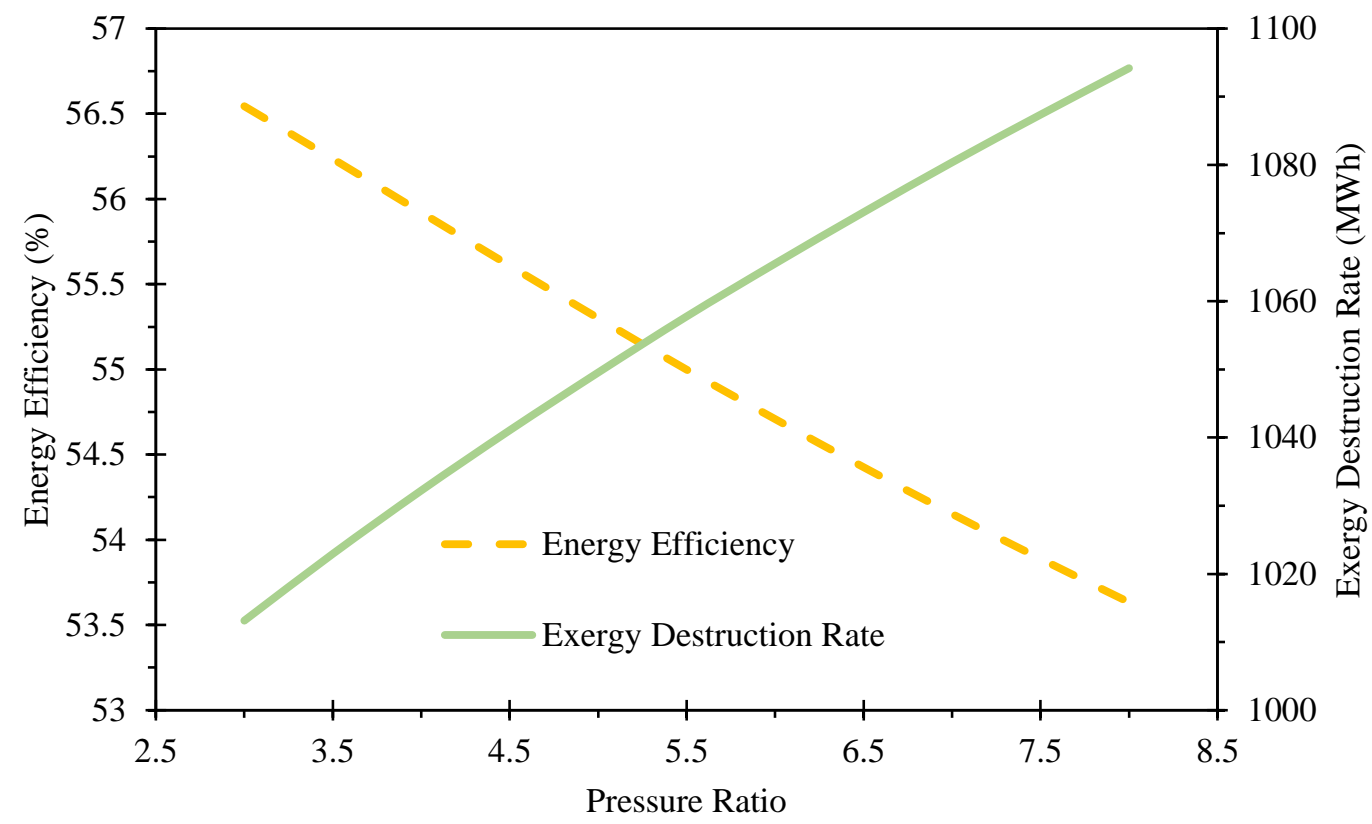

Figure 6 Effects of pressure ratio of each compression/expansion stage on the performance of the integrated system.

The influences of the heliostat numbers on the fuel consumption and power generation of the system are shown in Fig. 7. When the heliostat numbers are increased from 300 to 850, the daily generated power increases from 798.1 to $876 \mathrm{MWh}$, while the daily fuel consumption is reduced from 65.1 to 48.9 tons, respectively. This is because when the number of heliostats increases, the temperature of the air leaving heater 2 is increased drastically (from 391 to 695), and hence, the outlet temperature of the first stage gas turbine and its generated power are increased. On the other hand, when the inlet temperature of air entering the first stage of the gas turbine increases, by considering a constant value for the pressure ratio, its outlet temperature is also increased. This means that lower values of fuel will be required to heat up the air the chosen temperature. Therefore, the fuel consumption rate in the combustion chamber is decreased. 


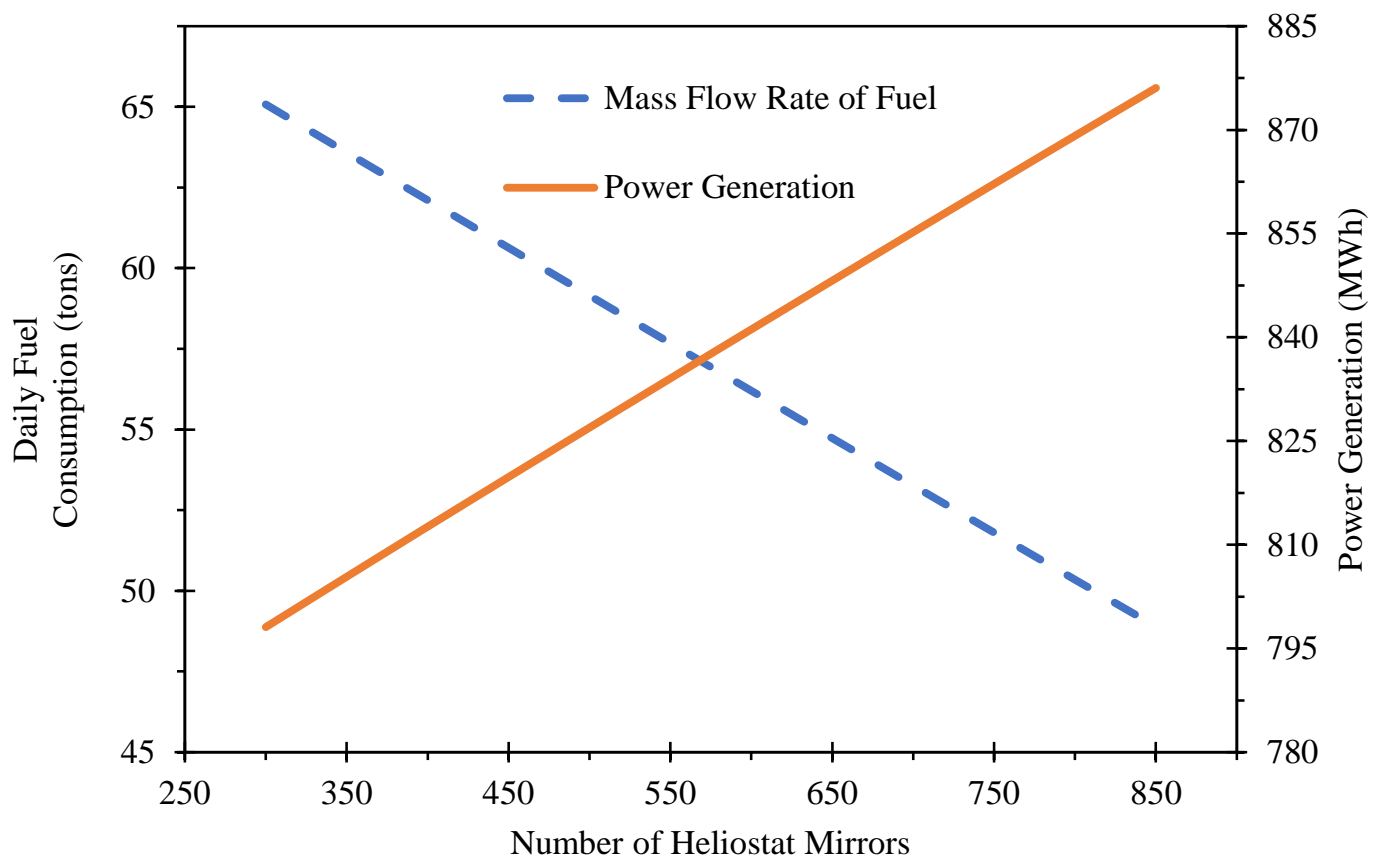

Figure 7 Influence of heliostat numbers on the integrated system performance.

The effect of the combustor outlet temperature on the performance of the integrated system is presented in Fig. 8. When the combustion chamber outlet temperature increases from 1,000 to 1,400 , the daily power generated by the integrated system increases from 748 to $1,016.1 \mathrm{MWh}$, and energy efficiency increases from $55.1 \%$ to $55.6 \%$. As the combustor outlet temperature increases, the power output of the GT cycle increases. This causes an increase in the outlet temperature of the gas turbine which in turn results in an increase in power generation of the liquefied air power cycle and TEG unit. To be more specific, with the same amount of increase in the GTIT, the power generated by the TEG, liquefied air power cycle, and GT cycle are increased from 36, 183.2, and 527.2 MWh to 112, 260, and 642.4 MWh, respectively. 


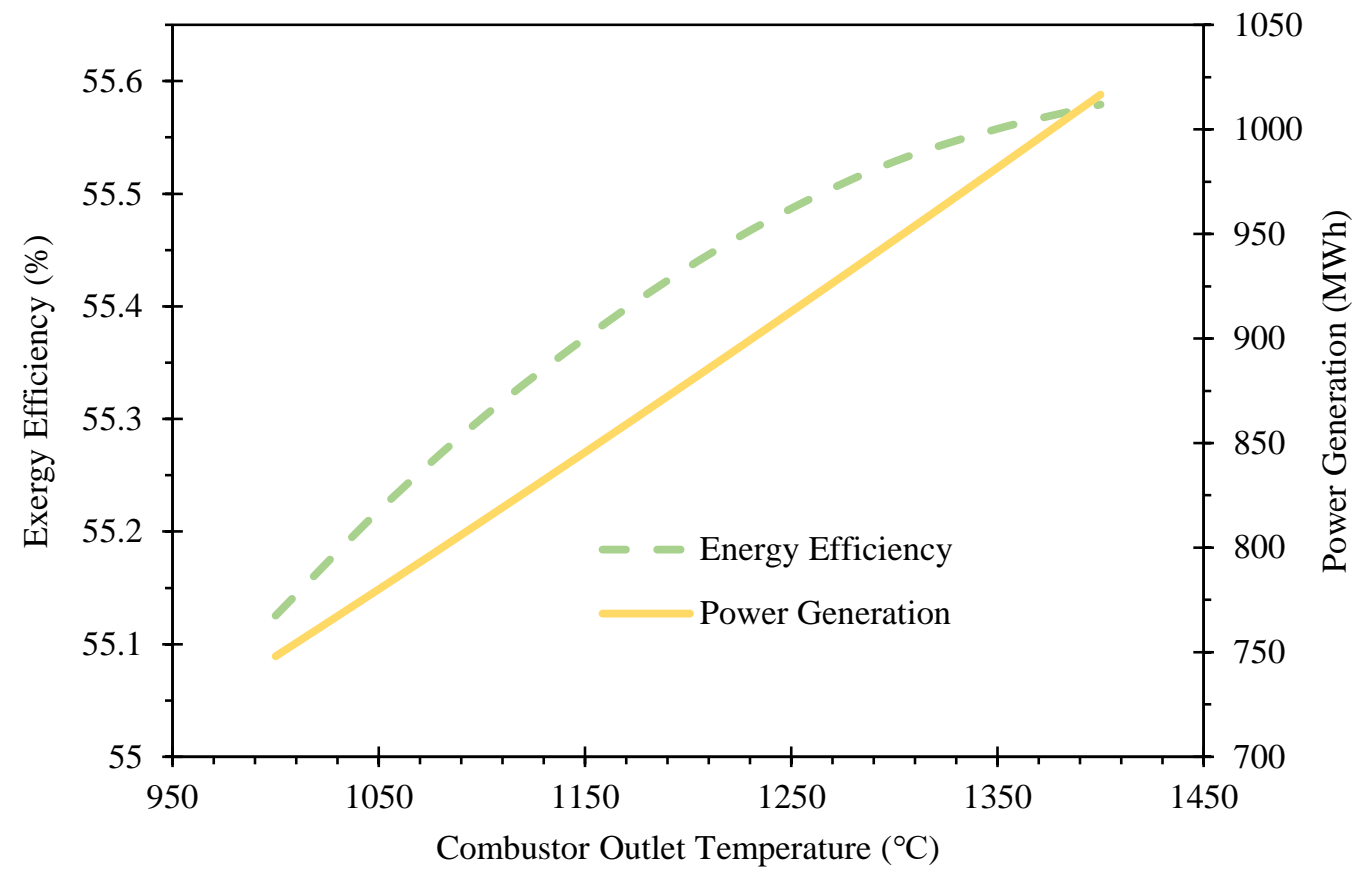

Figure 8 Influence of combustor outlet temperature on integrated system performance.

The influences of liquefied air power cycle turbine inlet pressure on the exergy destruction rate and generated power of the proposed system are presented in Fig. 9. As the inlet pressure of the liquefied air power cycle turbine increases from 5,000 to 18,000, the daily generated power increases from 735.9 to $821.2 \mathrm{MWh}$, while the exergy destruction of the system is reduced from 1,348.4 to 1,015.9 MWh, respectively. As the turbine inlet pressure increases, more heat is required in the liquefied air power cycle, and therefore, the temperature of flue gas leaving heater 3 is decreased. This translates into a more efficient consumption of the exergy content of the flue gas before discharging it to the atmosphere. 


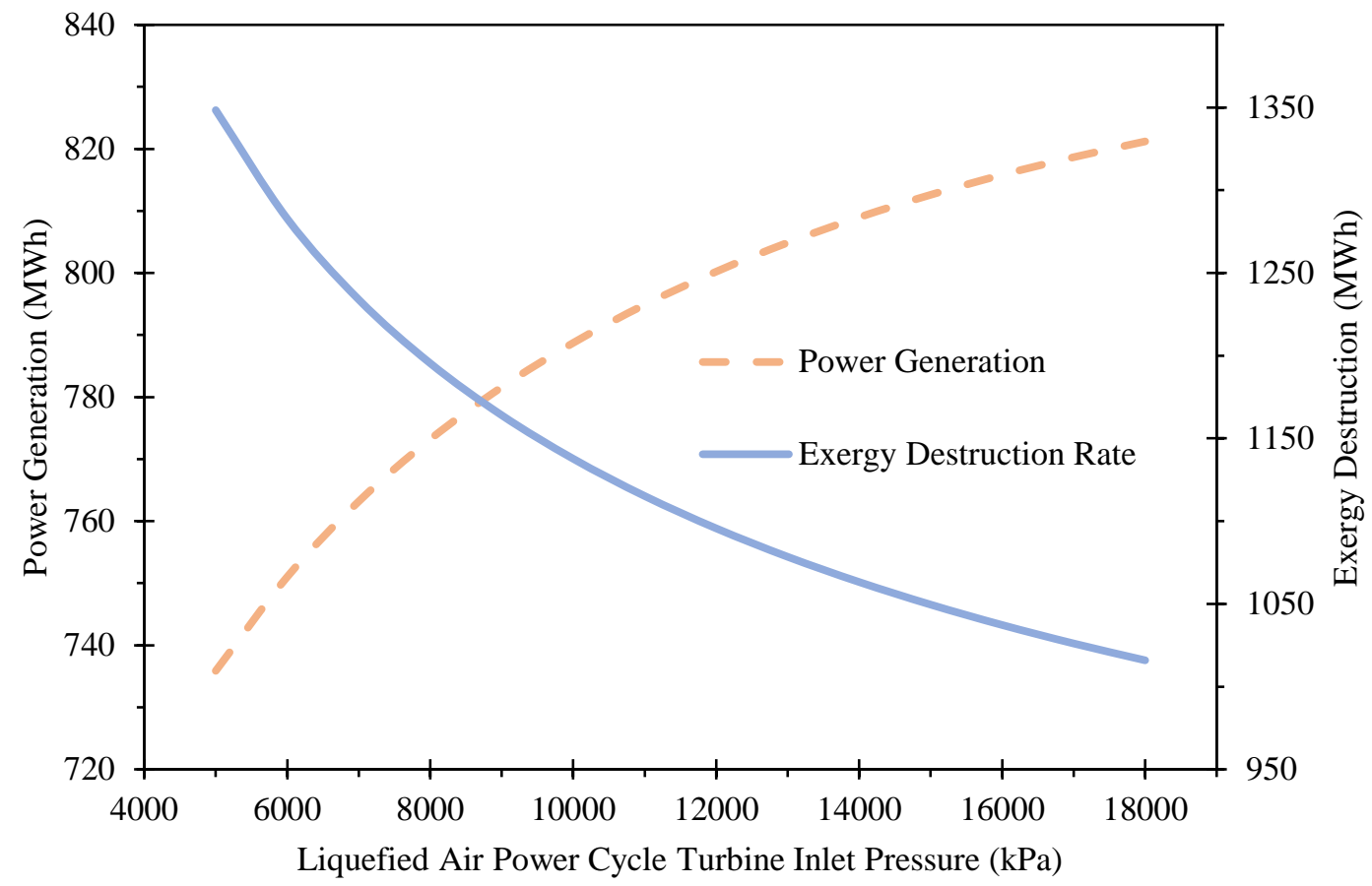

Figure 9 Influence of liquefied air power cycle turbine inlet pressure on integrated system performance.

The effects of LNG pump outlet pressure on the power generation and normalized $\mathrm{CO}_{2}$ emissions of the cycle are presented in Fig. 10. The system is optimized at the LNG pump outlet pressure of $12,000 \mathrm{kPa}$. The optimum results of daily power generation and normalized $\mathrm{CO}_{2}$ emissions are $815.8 \mathrm{MW}$ and $209 \mathrm{~kg} \mathrm{CO} / \mathrm{MWh}$, respectively. The liquefied air power cycle condenser temperature is dependent on the temperature of natural gas at the outlet of the LNG pump. The best performance of the liquefied air power cycle, and hence the integrated system is achieved at the LNG pump outlet pressure of $12000 \mathrm{kPa}$. 


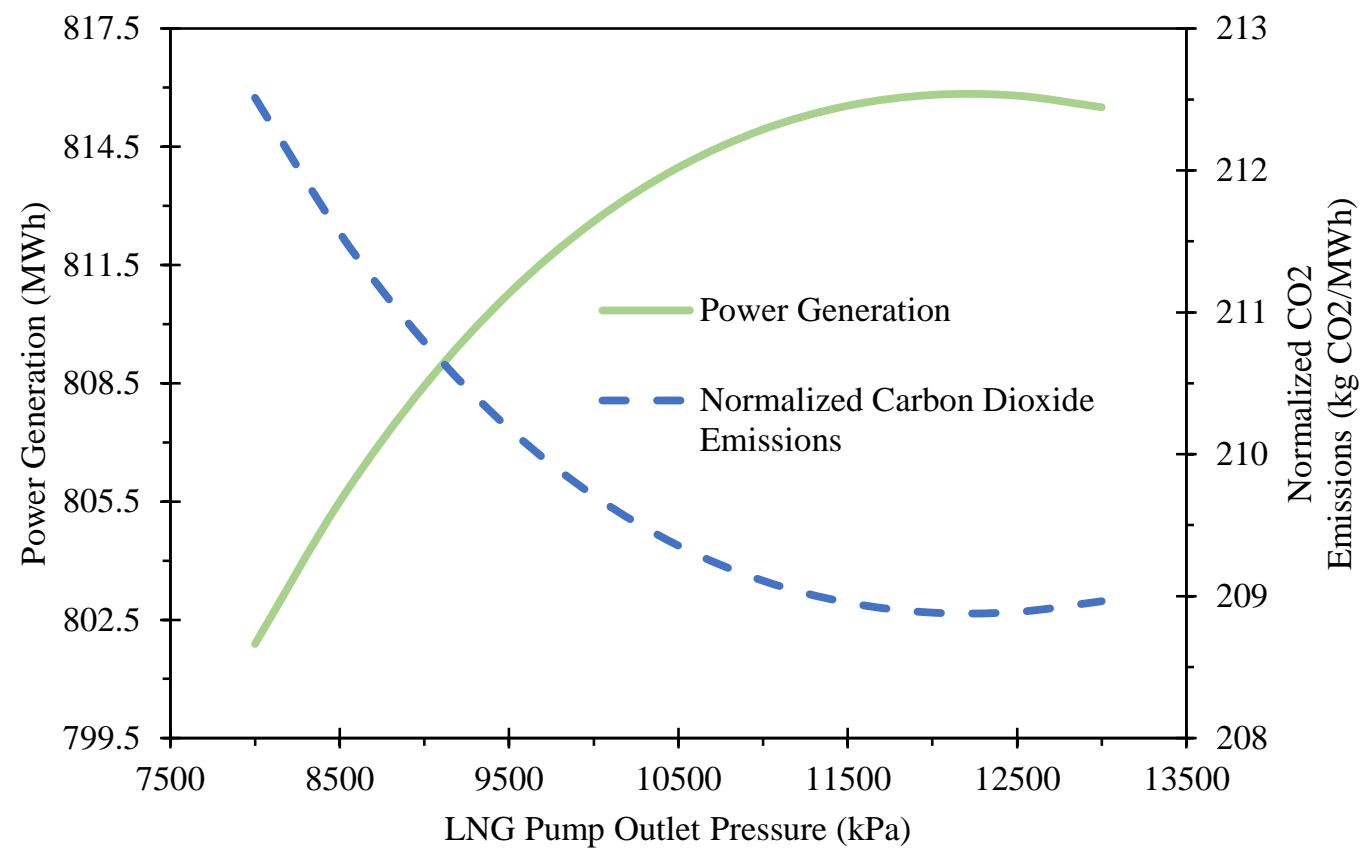

Figure 10 Effects of LNG pump outlet pressure on the integrated system performance.

\section{Comparison with past studies}

The energetic and exergetic efficiencies of the integrated solar-assisted CAES system are compared with those presented previously in Table 8. Past studies selected for comparison include a trigeneration CAES plant with absorption chiller and parabolic trough collectors [43], an advanced CAES system integrated with organic Rankine cycle/Kalina cycle for the waste heat recovery purposes [44], a hybrid CAES system which uses both wind and solar energies [45], another hybrid CAES integrated with solar PV panels, batteries, and fuel cell [46], and an integrated CAES system with waste heat recovery and wind farm [47]. It is shown that the integrated system proposed in this study has a better performance in terms of energy and exergy.

Table 8 A comparison between the present CAES system and past studies.

\begin{tabular}{|c|c|c|c|c|c|}
\hline Study & Subsystems & $\begin{array}{l}\text { Compressors } \\
\text { and turbine } \\
\text { efficiencies } \\
(\%)\end{array}$ & $\begin{array}{l}\text { Energy } \\
\text { efficiency } \\
(\%)\end{array}$ & $\begin{array}{l}\text { Exergy } \\
\text { Efficiency } \\
(\%)\end{array}$ & $\begin{array}{l}\mathrm{LCOE} \\
(\phi / \mathrm{kWh})\end{array}$ \\
\hline $\begin{array}{l}\text { Present } \\
\text { study }\end{array}$ & $\begin{array}{l}\text { CAES, } \\
\text { regasification, SNG } \\
\text { tower, and liquid air } \\
\text { power cycle }\end{array}$ & 80 and 86 & 54.9 & 46.9 & 12.4 \\
\hline $\begin{array}{l}\text { Wang et al. } \\
\text { [43] }\end{array}$ & $\begin{array}{l}\text { CAES, solar collector, gas } \\
\text { turbine, and absorbtion } \\
\text { chiller }\end{array}$ & 86 and 88 & 53.1 & 45.4 & - \\
\hline
\end{tabular}




\begin{tabular}{|l|l|l|l|l|l|}
\hline $\begin{array}{l}\text { Soltani et al. } \\
{[44]}\end{array}$ & $\begin{array}{l}\text { CAES, low and high } \\
\text { temperature } \\
\text { storage, Kalina cycle, and } \\
\text { ORC }\end{array}$ & 58 and 85 & $50.7-51.2$ & $44.2-44.6$ & - \\
\hline $\begin{array}{l}\text { Chen et al. } \\
{[45]}\end{array}$ & $\begin{array}{l}\text { CAES, wind farm, and } \\
\text { solar collector }\end{array}$ & $\sim 90$ & 46.5 & - & - \\
\hline $\begin{array}{l}\text { Sadeghi and } \\
\text { Askari [46] }\end{array}$ & $\begin{array}{l}\text { CAES, TES, fuel cell, } 82 \text { and } 86 \\
\text { solar PV pannels, battery, } \\
\text { and water reservoir }\end{array}$ & 55 & - & 15.6 \\
\hline $\begin{array}{l}\text { Meng et al. } \\
\text { [47] }\end{array}$ & $\begin{array}{l}\text { CAES, waste heat } \\
\text { recovery unit, and wind } \\
\text { farm }\end{array}$ & 54 and 90 & 53.3 & - & $5-8$ \\
\hline
\end{tabular}

\section{Conclusions}

A novel solar-assisted integrated compressed air energy storage system is designed and analyzed in this paper. The proposed system comprises an air compression unit, thermal oil loop, gas turbine, solar power tower with thermal energy storage, liquefied natural gas regasification unit, thermoelectric generator, and liquefied air power cycle. The performance of the energy storage system is studied through exergy, energy, and economic analyses. A parametric study is performed to examine the effects of varying major parameters on the performance of the proposed integrated system. Major findings are presented as follows:

- The energy and exergy efficiencies of the integrated system are $55.3 \%$ and $46.4 \%$, respectively.

- $\quad 356 \mathrm{MWh}$ of power is consumed during charging mode and 797.6 MWh of power is generated during the discharging mode. This indicates that the energy storage system proposed here uses less than half of the power it produces during the discharging mode with the aid of solar energy and cold waste heat of liquefied natural gas.

- 5,539.5 tons of LNG is required per day for the integrated plant to be operational for 8 hours of discharging.

- Excess air is used in the combustion process to reduce fuel consumption and improve the performance of the energy storage system. It is observed that a daily value of 63.4 tons of fuel is required during the discharging mode of the system.

- Dynamic modelling of solar subsystem showed that the solar heat input to the heliostat mirrors is maximized at 11 A.M. with a value of $39.1 \mathrm{MW}$. The abosrbed heat by the molten salt at this time is $23.74 \mathrm{MW}$. Therefore, the solar subsystem efficiency is evaluated as $60.7 \%$ at 11 A.M.

- The normalized carbon dioxide emissions of the system are $210.1 \mathrm{~kg} \mathrm{CO} / \mathrm{MWh}$. The current system is three times less harmful to the environment compared to the carbon impact of Iranian national grid.

- The highest contributors to the overall exergy destruction of the system are the combustion chamber, heater 4, solar tower, recuperator, and heliostats with values of 370.9, 164.9, 
89.8, 81.5, and 69.7 MWh, respectively. Therefore, to improve environmental sustainability, these components require further investigation in future research.

- Overall exergy destruction of the system during both charging and discharging phases are calculated as 1,035.2 MWh.

- Enhancing the pressure ratio of the compressors will reduce the performance of the plant, while increasing the combustion chamber outlet temperature will improve it. Also, the normalized carbon dioxide emissions of the plant are minimized at LNG pump outlet pressure of $12 \mathrm{MPa}$. Increasing the number of heliostat mirrors from 300 to 850 will increase the daily power generation of the plant 798.1 to $876 \mathrm{MWh}$, while the daily fuel consumption is reduced from 65.1 to 48.9 tons.

- Economic analysis indicated that the levelized cost of electricity is $12.4 \phi / \mathrm{kWh}$.

\section{References}

[1] Turkson, C., Acquaye, A., Liu, W., Papadopoulos, T. Sustainability assessment of energy production: A critical review of methods, measures, and issues. Journal of Environmental Management, 264, 110464, 2020.

[2] Suberu, M. Y., Mustafa, M. W., Bashir, N. Energy storage systems for renewable energy power sector integration and mitigation of intermittency. Renewable and Sustainable Energy Reviews, 35, 499-514, 2014.

[3] Palmer, G., Floyd, J. Energy Storage and Civilization: A Systems Approach (Vol. 40). Springer Nature, 2020.

[4] Akinyele, D. O., Rayudu, R. K. Review of energy storage technologies for sustainable power networks. Sustainable Energy Technologies and Assessments, 8, 74-91, 2014.

[5] Alami, A. H., Aokal, K., Abed, J., Alhemyari, M. Low pressure, modular compressed air energy storage (CAES) system for wind energy storage applications. Renewable Energy, 106, 201211, 2017.

[6] Koohi-Fayegh, S., Rosen, M. A. A review of energy storage types, applications and recent developments. Journal of Energy Storage, 27, 101047, 2020.

[7] Budt, M., Wolf, D., Span, R., Yan, J. A review on compressed air energy storage: Basic principles, past milestones and recent developments. Applied Energy, 170, 250-268, 2016.

[8] Rufer, A. Energy storage: systems and components. CRC Press, 2017.

[9] Liu, H., He, Q., Saeed, S. B. Thermodynamic analysis of a compressed air energy storage system through advanced exergetic analysis. Journal of Renewable and Sustainable Energy, 8(3), 034101, 2016.

[10] Krawczyk, P., Szabłowski, Ł., Karellas, S., Kakaras, E., Badyda, K. Comparative thermodynamic analysis of compressed air and liquid air energy storage systems. Energy, 142, 4654, 2018.

[11] Peng, H., Yang, Y., Li, R., Ling, X. Thermodynamic analysis of an improved adiabatic compressed air energy storage system. Applied Energy, 183, 1361-1373, 2016.

[12] Liu, J. L., Wang, J. H. A comparative research of two adiabatic compressed air energy storage systems. Energy Conversion and Management, 108, 566-578, 2016. 
[13] Zhao, P., Wang, J., Dai, Y. Thermodynamic analysis of an integrated energy system based on compressed air energy storage (CAES) system and Kalina cycle. Energy Conversion and Management, 98, 161-172, 2015.

[14] Szablowski, L., Krawczyk, P., Badyda, K., Karellas, S., Kakaras, E., Bujalski, W. Energy and exergy analysis of adiabatic compressed air energy storage system. Energy, 138, 12-18, 2017.

[15] Carriveau, R., Ebrahimi, M., Ting, D. S. K., McGillis, A. Transient thermodynamic modeling of an underwater compressed air energy storage plant: Conventional versus advanced exergy analysis. Sustainable Energy Technologies and Assessments, 31, 146-154, 2019.

[16] Sadreddini, A., Fani, M., Aghdam, M. A., Mohammadi, A. Exergy analysis and optimization of a CCHP system composed of compressed air energy storage system and ORC cycle. Energy conversion and management, 157, 111-122, 2018.

[17] Moran, M. J., Bailey, M. B., Boettner, D. D., Shapiro, H. N. Fundamentals of engineering thermodynamics. 9th Edition, Wiley, 2018.

[18] Ghorbani, B., Miansari, M., Zendehboudi, S., Hamedi, M. H. Exergetic and economic evaluation of carbon dioxide liquefaction process in a hybridized system of water desalination, power generation, and liquefied natural gas regasification. Energy Conversion and Management, 205, 112374, 2020.

[19] Xue, F., Chen, Y., Ju, Y. A review of cryogenic power generation cycles with liquefied natural gas cold energy utilization. Frontiers in Energy, 10(3), 363-374, 2016.

[20] Zhang, Y., Yao, E., Zhang, X., \& Yang, K. Thermodynamic analysis of a novel compressed carbon dioxide energy storage system with low-temperature thermal storage. International Journal of Energy Research. 44, 6531-6554, 2020.

[21] Saputro, E. A., Farid, M. M. Performance of a small-scale compressed air storage (CAS). International Journal of Energy Research, 43(12), 6233-6242, 2019.

[22] Karapekmez, A., Dincer, I., Javani, N. Development of a new integrated energy system with compressed air and heat storage options. Journal of Energy Storage, 32, 101955, 2020

[23] Lee, I., Park, J., Moon, I. Conceptual design and exergy analysis of combined cryogenic energy storage and LNG regasification processes: Cold and power integration. Energy, 140, 106115, 2017.

[24] Wang, Z., Xiong, W., Carriveau, R., Ting, D. S. K., Wang, Z. Energy, exergy, and sensitivity analyses of underwater compressed air energy storage in an island energy system. International Journal of Energy Research, 43(6), 2241-2260, 2019.

[25] Wang, P., Zhao, P., Lai, Y., Wang, J., Dai, Y. Performance comparison of different combined heat and compressed air energy storage systems integrated with organic Rankine cycle. International Journal of Energy Research, 43(14), 8410-8425, 2019.

[26] Hao, Y., He, Q., Liu, W., Pan, L., Oldenburg, C. M. Thermodynamic analysis of a novel fossil-fuel-free energy storage system with a trans-critical carbon dioxide cycle and heat pump. International Journal of Energy Research, 44(10), 7924-7937, 2020.

[27] Ji, W., Zhou, Y., Sun, Y., Zhang, W., An, B., Wang, J. Thermodynamic analysis of a novel hybrid wind-solar-compressed air energy storage system. Energy Conversion and Management, 142, 176-187, 2017. 
[28] Mohammadi, A., Mehrpooya, M. Exergy analysis and optimization of an integrated micro gas turbine, compressed air energy storage and solar dish collector process. Journal of Cleaner Production, 139, 372-383, 2016.

[29] Yang, C., Wang, X., Huang, M., Ding, S., Ma, X. Design and simulation of gas turbine-based CCHP combined with solar and compressed air energy storage in a hotel building. Energy and Buildings, 153, 412-420, 2017.

[30] Roushenas, R., Razmi, A. R., Soltani, M., Torabi, M., Dusseault, M. B., Nathwani, J. Thermoenvironmental analysis of a novel cogeneration system based on solid oxide fuel cell and compressed air energy storage coupled with turbocharger. Applied Thermal Engineering, 115978, 2020.

[31] Mohammadi, A., Ahmadi, M. H., Bidi, M., Joda, F., Valero, A., Uson, S. Exergy analysis of a Combined Cooling, Heating and Power system integrated with wind turbine and compressed air energy storage system. Energy Conversion and Management, 131, 69-78, 2017.

[32] Vignarooban, K., Xu, X., Arvay, A., Hsu, K., Kannan, A. M. Heat transfer fluids for concentrating solar power systems-a review. Applied Energy, 146, 383-396, 2015.

[33] Borgnakke, C., Sonntag, R. E. Fundamentals of Thermodynamics. 10th Edition, Wiley, 2019. [34] Xu, C., Wang, Z., Li, X., Sun, F. Energy and exergy analysis of solar power tower plants. Applied Thermal Engineering, 31(17-18), 3904-3913, 2011.

[35] Ziapour, B. M., Saadat, M., Palideh, V., \& Afzal, S. Power generation enhancement in a salinity-gradient solar pond power plant using thermoelectric generator. Energy conversion and management, 136, 283-293, 2017.

[36] An, X., Cheng, J., Zhang, P., Tang, Z., Wang, J. Determination and evaluation of the thermophysical properties of an alkali carbonate eutectic molten salt. Faraday discussions, 190, 327-338, 2016.

[37] Bejan, A., Tsatsaronis, G., Moran, M. J. Thermal design and optimization. John Wiley \& Sons, 1995.

[38] Couper, J. R., Penney, W. R., Fair, J. R. Chemical Process Equipment-Selection and Design (Revised 2nd Edition). Gulf Professional Publishing, 2009.

[39] Mosaffa, A. H., Farshi, L. G., Ferreira, C. I., Rosen, M. A. Exergoeconomic and environmental analyses of $\mathrm{CO} 2 / \mathrm{NH} 3$ cascade refrigeration systems equipped with different types of flash tank intercoolers. Energy Conversion and Management, 117, 442-453, 2016.

[40] Dincer, I., Rosen, M. A., Ahmadi, P. Optimization of energy systems. New York: Wiley, 2017.

[41] Zhuang, X., Xu, X., Liu, W., Xu, W. LCOE Analysis of Tower Concentrating Solar Power Plants Using Different Molten-Salts for Thermal Energy Storage in China. Energies, 12(7), 1394, 2019.

[42] Habibollahzade, A., Gholamian, E., Ahmadi, P., Behzadi, A. Multi-criteria optimization of an integrated energy system with thermoelectric generator, parabolic trough solar collector and electrolysis for hydrogen production. International journal of hydrogen energy, 43(31), 1414014157, 2018.

[43] Wang, X., Yang, C., Huang, M., Ma, X. Multi-objective optimization of a gas turbine-based CCHP combined with solar and compressed air energy storage system. Energy Conversion and Management, 164, 93-101, 2018. 
[44] Soltani, M., Nabat, M. H., Razmi, A. R., Dusseault, M. B., Nathwani, J. A comparative study between ORC and Kalina based waste heat recovery cycles applied to a green compressed air energy storage (CAES) system. Energy Conversion and Management, 222, 113203, 2020.

[45] Chen, J., Liu, W., Jiang, D., Zhang, J., Ren, S., Li, L., Li, X., Shi, X. Preliminary investigation on the feasibility of a clean CAES system coupled with wind and solar energy in China. Energy, 127, 462-478, 2017.

[46] Sadeghi, S., Askari, I. B. Prefeasibility techno-economic assessment of a hybrid power plant with photovoltaic, fuel cell and Compressed Air Energy Storage (CAES). Energy, 168, 409-424, 2019.

[47] Meng, H., Wang, M., Olumayegun, O., Luo, X., Liu, X. Process design, operation and economic evaluation of compressed air energy storage (CAES) for wind power through modelling and simulation. Renewable Energy, 136, 923-936, 2019. 